\title{
Hyperboloidal Einstein-matter evolution and tails for scalar and Yang-Mills fields
}

\author{
Oliver Rinne $^{1}$ and Vincent Moncrief ${ }^{2}$ \\ ${ }^{1}$ Max Planck Institute for Gravitational Physics (Albert Einstein Institute), Am \\ Mühlenberg 1, 14476 Potsdam, Germany \\ ${ }^{2}$ Department of Mathematics and Department of Physics, Yale University, New \\ Haven, CT 06520, USA \\ E-mail: oliver.rinne@aei.mpg.de, vincent.moncrief@yale.edu
}

\begin{abstract}
We show how matter can be included in a constrained ADM-like formulation of the Einstein equations on constant mean curvature surfaces. Previous results on the regularity of the equations at future null infinity are unaffected by the addition of matter with tracefree energy-momentum tensor. Two examples are studied in detail, a conformally coupled scalar field and a YangMills field. We first derive the equations under no symmetry assumptions and then reduce them to spherical symmetry. Both sectors (gravitational and sphaleron) of the spherically symmetric Yang-Mills field are included. We implement this scheme numerically in order to study late-time tails of scalar and Yang-Mills fields coupled to the Einstein equations. We are able to evolve spacetimes that disperse to flat space, accrete onto a given black hole or collapse to a black hole from regular initial data. The sphaleron sector of Yang-Mills is found to exhibit some nontrivial gauge dynamics.
\end{abstract}

\section{Introduction}

Most current numerical relativity codes are based on the Cauchy formulation of general relativity and evolve spacetime on spacelike slices approaching spacelike infinity, truncated at some finite distance. At the resulting artificial timelike boundary, boundary conditions must be imposed that yield a well-posed initialboundary value problem and are compatible with the constraint equations. In addition, for evolutions of isolated systems, gravitational radiation should pass through the boundary without causing spurious reflections, i.e. the boundary conditions should be absorbing. Considerable progress has been made recently with the construction and implementation of boundary conditions for the Einstein equations (see [1 for a recent review article). A fundamental problem remains, however. In general relativity there is no well-defined flux of gravitational radiation at a finite distance upon which absorbing boundary conditions could be based. At best one may appeal to linearised theory. Gravitational radiation is only well defined at future null infinity $\mathscr{I}^{+}$. Thus a far more elegant solution to the outer boundary problem is to include $\mathscr{I}^{+}$in the numerical domain.

We follow Penrose's approach 2 and apply a conformal transformation to the spacetime metric, combined with a compactifying coordinate transformation that maps an asymptotically flat spacetime to a finite domain. In [3] we developed 
an ADM-like 4 formulation of the vacuum Einstein equations on constant mean curvature (CMC) surfaces. These are spacelike but approach future null infinity instead of spacelike infinity; thus one solves a hyperboloidal initial value problem. (Note that hyperboloidal surfaces are not Cauchy surfaces; we only obtain the part of spacetime to the future of the initial hyperboloidal surface.) Since the Ricci tensor is not conformally invariant, the Einstein equations contain inverse powers of the conformal factor that are singular at $\mathscr{I}^{+}$. However, in 3 we showed how the formally singular terms in the ADM evolution equations can be evaluated at $\mathscr{I}^{+}$in a regular way provided the constraints hold and $\mathscr{I}^{+}$is shear free. In [5] this scheme was implemented numerically for axisymmetric spacetimes. Long-term stable evolutions of a gravitationally perturbed Schwarzschild black hole were obtained and the Bondi news function describing the gravitational radiation emitted by the system was evaluated at $\mathscr{I}^{+}$.

Before continuing we briefly review other hyperboloidal evolution schemes and associated numerical studies. The oldest and, arguably, mathematically best understood formulation are the regular conformal field equations due to Friedrich 6. These form a symmetric hyperbolic system of partial differential equations that contain the Einstein equations as well as evolution equations for the Weyl curvature arising from the Bianchi identities. The equations have the remarkable property that they are manifestly regular up to $\mathscr{I}^{+}$. There have been various attempts at numerical evolutions based on these equations (see the review articles [7, 8, 9]). With a view to the applications considered in the present paper, we mention in particular the studies of spherically symmetric scalar field collapse by Hübner [10, 11]. Recently a number of formulations have been suggested that are based more directly on the Einstein equations, as in our approach. Zenginoğlu [12] developed a formulation based on generalised harmonic gauge combined with a suitable choice of gauge source functions at $\mathscr{I}^{+}$. Bardeen, Sarbach and Buchman 13 derived a tetrad formulation of the Einstein equations on CMC slices. First numerical results on initial data for single and binary black holes were presented in 14,15 .

In this paper we return to the constrained ADM formulation on CMC slices developed in [3] and extend it to include matter sources. The motivation for this derives partly from the fact that we wanted to study the late-time behaviour of perturbed black hole spacetimes in the context of the full (rather than linearised) Einstein equations, including future null infinity. In [5] we correctly reproduced the quasi-normal mode radiation emitted by a perturbed black hole but due to limited numerical resolution we were unable to resolve the power-law tail expected at later times. Therefore, in order to see if our method is suitable to study these phenomena, we decided to take one step back and consider spherically symmetric spacetimes, which are computationally less expensive to evolve. Because of Birkhoff's theorem, matter is needed in order to have nontrivial dynamics in spherical symmetry. How to include matter in a hyperboloidal Einstein evolution scheme is an interesting problem in its own right.

At late times matter fields as well as gravitational perturbations on flat space and black hole spacetimes typically decay polynomially in time, a phenomenon often referred to as Price's law [16. This power-law tail is caused by the backscatter off the curved background spacetime and/or by the nonlinearity of the matter fields. It plays an important role in trying to prove stability of black hole spacetimes and the cosmic censorship conjecture [17. At $\mathscr{I}^{+}$the fields generally decay at a slower rate than at any finite distance, although the closer an observer is to $\mathscr{I}^{+}$, the longer the 
measured decay rate stays close to the value corresponding to $\mathscr{I}^{+}$before it ultimately approaches the faster finite-distance decay. It can be argued [18] that the decay rate at $\mathscr{I}^{+}$is the relevant one for observers in the astrophysical zone [19, in which the distance to the source of radiation is very large compared to the time during which the signal is observable.

For scalar fields the tail decay rates can be predicted from linear perturbation theory 20, 21] and have been confirmed numerically many times. We mention two recent studies that both include $\mathscr{I}^{+}$in the numerical domain. Pürrer, Husa and Aichelburg [18] evolved the spherically symmetric Einstein-scalar field system in Bondi coordinates and determined the power-law tails in subcritical evolutions that disperse to flat space. Bondi coordinates cannot penetrate horizons so the decay of the field at the horizon of a black hole could not be studied. Zenginoğlu evolved the spherically symmetric scalar wave equation on a fixed background spacetime (taken to be either Minkowski or Schwarzschild), the test field approximation. He used a hyperboloidal foliation of spacetime that covers part of the black hole interior as well. In the present paper we evolve the coupled Einstein-scalar field equations on hyperboloidal slices reaching out to $\mathscr{I}^{+}$. In particular, we are able to study the decay of the field at the horizon of a black hole formed in gravitational collapse.

For Yang-Mills fields the prediction from linear perturbation theory turns out to be incorrect: the nonlinearity of the field causes a slower decay 22. Similarly to the scalar field, the Einstein-Yang-Mills system was evolved in Bondi coordinates in 23. and in the test field approximation on hyperboloidal slices in 24. We shall compare our results with those studies.

This paper is organised as follows. In section 2 we extend our hyperboloidal Einstein evolution scheme [3] to include matter sources and we re-examine the question of regularity at $\mathscr{I}^{+}$. Two matter models are studied in detail in section 3 a conformally coupled scalar field and a Yang-Mills field. In section 4 we reduce our formulation to spherical symmetry. The numerical implementation of this system is described in section 5 and our results on power-law tails are presented in section 6 Finally we conclude and discuss some directions for future work in section 7 Some useful identities for conformal transformations and $3+1$ decompositions are collected in Appendix A

\section{General formalism}

In this section we consider general matter subject to the condition that its energymomentum tensor be tracefree. We extend the conformal ADM formulation of the Einstein equations derived in 3 to include the corresponding matter source terms. The question of regularity at future null infinity is re-examined.

\subsection{Matter in the conformal setting}

We use the notation and conventions of [3]. The spacetime metric ${ }^{(4)} g_{\mu \nu}$ is written as

$$
{ }^{(4)} g_{\mu \nu}=\Omega^{-2(4)} \gamma_{\mu \nu},
$$

where ${ }^{(4)} \gamma_{\mu \nu}$ is the conformal spacetime metric and $\Omega$ the conformal factor. Consider now matter given by an energy-momentum tensor $T_{\mu \nu}$. We introduce a conformally rescaled energy-momentum tensor $\tilde{T}_{\mu \nu}$ via

$$
T_{\mu \nu}=\Omega^{2} \tilde{T}_{\mu \nu} .
$$


The energy-momentum conservation equations transform as 25]

$$
{ }^{(4)} \gamma^{\mu \nu(4)} \tilde{\nabla}_{\mu} \tilde{T}_{\nu \rho}=\Omega^{-4(4)} g^{\mu \nu}\left({ }^{(4)} \nabla_{\mu} T_{\nu \rho}-\Omega^{-1(4)} \nabla_{\rho} \Omega T_{\mu \nu}\right),
$$

where ${ }^{(4)} \nabla$ denotes the covariant derivative of ${ }^{(4)} g$ and ${ }^{(4)} \tilde{\nabla}$ the covariant derivative of ${ }^{(4)} \gamma$. The first term on the right-hand side of (3) vanishes by energy-momentum conservation. If the energy-momentum tensor is tracefree,

$$
{ }^{(4)} g^{\mu \nu} T_{\mu \nu}=0,
$$

then the second term on the right-hand side of (3) also vanishes and the energymomentum conservation equations reduce to

$$
{ }^{(4)} \gamma^{\mu \nu(4)} \tilde{\nabla}_{\mu} \tilde{T}_{\nu \rho}=0 \text {. }
$$

Assuming that $\tilde{T}_{\mu \nu}$ is itself regular at $\mathscr{I}^{+}$, which it is if its basic fields satisfy conformally regular field equations (as in the matter models considered in section (3), then (5) is manifestly regular at future null infinity $\mathscr{I}^{+}$. For this reason we will restrict ourselves to matter with tracefree energy-momentum tensor.

Examples of matter models satisfying this condition include the conformally coupled scalar field (section 3.1), Maxwell and more generally Yang-Mills fields (section 3.2) and the radiation fluid, i.e. a perfect fluid with equation of state $p=\frac{1}{3} \rho$ (see also [26]). There are of course many matter models that do not satisfy (44). Typically however, these are considered to be less radiative. For example, a massive scalar field is known to fall off faster than any power of radius towards $\mathscr{I}^{+}$[27]. If the matter remains bounded away from $\mathscr{I}^{+}$, as is expected in many situations of astrophysical interest, then of course there is no harm in using the singular matter field equations away from $\mathscr{I}^{+}$.

\subsection{Conformal ADM reduction of the Einstein equations with matter sources}

As in [3] we decompose the physical and conformal spacetime metric in $3+1$ form,

$$
\begin{aligned}
& { }^{(4)} g=-N^{2} \mathrm{~d} t^{2}+g_{i j}\left(\mathrm{~d} x^{i}+X^{i} \mathrm{~d} t\right)\left(\mathrm{d} x^{j}+X^{j} \mathrm{~d} t\right), \\
& { }^{(4)} \gamma=-\tilde{N}^{2} \mathrm{~d} t^{2}+\gamma_{i j}\left(\mathrm{~d} x^{i}+X^{i} \mathrm{~d} t\right)\left(\mathrm{d} x^{j}+X^{j} \mathrm{~d} t\right),
\end{aligned}
$$

where the physical and conformal lapse are related via $N=\Omega^{-1} \tilde{N}$, the physical and conformal spatial metric via $g=\Omega^{-2} \gamma$, and $X^{i}$ serves both as physical and conformal shift. The unit timelike normals to the $t=$ const hypersurfaces in physical and conformal spacetime are related by $n^{\mu}=\Omega \tilde{n}^{\mu}$.

The extrinsic curvature is defined as

$$
K_{i j}=-\frac{1}{2} \mathcal{L}_{n} g_{i j} .
$$

Our condition on the time slices is that their mean extrinsic curvature be constant,

$$
g^{i j} K_{i j} \equiv-K=\mathrm{const}
$$

with $K>0$ so that the slices approach future null infinity (hence our slightly awkward sign convention in (9)). We choose to work with the traceless part of the ADM momentum, which is given in terms of the extrinsic curvature by

$$
\pi^{\text {tr } i j}=-\mu_{g}\left(g^{i k} g^{j l}-\frac{1}{3} g^{i j} g^{k l}\right) K_{k l},
$$

where $\mu_{g}=\sqrt{\operatorname{det}\left(g_{i j}\right)}$. For later use we also define the conformal extrinsic curvature

$$
C_{i j}=-\frac{1}{2} \mathcal{L}_{\tilde{n}} \gamma_{i j} .
$$


Its trace $C \equiv \gamma^{i j} C_{i j}$ is related to the variable $\Gamma=-2 \tilde{N} C$ introduced in $[3]$.

Following [28] we define the following projections of the energy-momentum tensor,

$$
\rho \equiv n^{\mu} n^{\nu} T_{\mu \nu}, \quad J^{i} \equiv-g^{i \mu} n^{\nu} T_{\mu \nu}, \quad S_{i j} \equiv g_{i}{ }^{\mu} g_{j}{ }^{\nu} T_{\mu \nu},
$$

and similarly for the conformally rescaled energy-momentum tensor (2),

$$
\tilde{\rho} \equiv \tilde{n}^{\mu} \tilde{n}^{\nu} \tilde{T}_{\mu \nu}, \quad \tilde{J}^{i} \equiv-\gamma^{i \mu} \tilde{n}^{\nu} \tilde{T}_{\mu \nu}, \quad \tilde{S}_{i j} \equiv \gamma_{i}{ }^{\mu} \gamma_{j}{ }^{\nu} \tilde{T}_{\mu \nu} .
$$

Clearly these quantities are related by

$$
\rho=\Omega^{4} \tilde{\rho}, \quad J^{i}=\Omega^{5} \tilde{J}^{i}, \quad S_{i j}=\Omega^{2} \tilde{S}_{i j} .
$$

The Einstein equations are

$$
G_{\mu \nu}=\kappa T_{\mu \nu}
$$

with $\kappa=8 \pi$ in geometric units (i.e. Newton's constant and the speed of light $G=c=1)$. They split into evolution equations and constraints. The evolutions equations are (cf. equations (2.10), (2.21) and (2.22) in [3])

$$
\begin{aligned}
& \mathcal{L}_{\tilde{n}} \Omega=-\frac{1}{3}(K+\Omega C), \\
& \mathcal{L}_{\tilde{n}} \gamma_{i j}=2 \mu_{\gamma}^{-1} \gamma_{i k} \gamma_{j l} \pi^{\operatorname{tr} k l}-\frac{2}{3} \gamma_{i j} C, \\
& \mathcal{L}_{\tilde{n}} \pi^{\operatorname{tr} i j}=-2 \mu_{\gamma}^{-1} \gamma_{k l} \pi^{\operatorname{tr} i k} \pi^{\operatorname{tr} j l}-\frac{2}{3} \Omega^{-1} K \pi^{\operatorname{tr} i j} \\
& +\mu_{\gamma}\left[\tilde{N}^{-1} \tilde{\nabla}^{i} \tilde{\nabla}^{j} \tilde{N}-\tilde{R}^{i j}-2 \Omega^{-1} \tilde{\nabla}^{i} \tilde{\nabla}^{j} \Omega+\kappa \Omega^{2} \tilde{S}^{i j}\right]^{\text {tr }} .
\end{aligned}
$$

Here and in the following, indices on quantities carrying a tilde are to be raised and lowered with $\gamma, \operatorname{tr}$ denotes the traceless part w.r.t. $\gamma, \tilde{R}_{i j}$ is the Ricci tensor of $\gamma$, and $\mathcal{L}$ denotes the Lie derivative. Note that $\pi^{\text {tr } i j}$ is a tensor density of weight one and hence

$$
\mathcal{L}_{\tilde{n}} \pi^{\operatorname{tr} i j}=\tilde{N}^{-1}\left[\partial_{t} \pi^{\operatorname{tr} i j}-\left(X^{k} \pi^{\operatorname{tr} i j}\right)_{, k}+X^{i}{ }_{, k} \pi^{\operatorname{tr} k j}+X^{j}{ }_{, k} \pi^{\operatorname{tr} i k}\right] .(19
$$

The Hamiltonian and momentum constraints are (cf. equations (2.9) and (2.7) in [3])

$0=-4 \Omega \tilde{\nabla}^{j} \tilde{\nabla}_{j} \Omega+6 \gamma^{i j} \Omega_{, i} \Omega_{, j}-\Omega^{2} \tilde{R}-\frac{2}{3} K^{2}+\Omega^{2} \mu_{\gamma}^{-2} \gamma_{i k} \gamma_{j l} \pi^{\operatorname{tr} i j} \pi^{\operatorname{tr} k l}+2 \kappa \Omega^{4} \tilde{\rho}$,

$0=\tilde{\nabla}_{j}\left(\Omega^{-2} \pi^{\operatorname{tr} i j}\right)+\kappa \mu_{\gamma} \tilde{J}^{i}$.

Preservation of the CMC condition, $\partial_{t} K=0$, implies an elliptic equation for the conformal lapse (cf. equation (2.13) in [3]),

$$
\begin{aligned}
-\Omega^{2} \gamma^{i j} \tilde{\nabla}_{i} \tilde{\nabla}_{j} \tilde{N} & +3 \Omega \gamma^{i j} \tilde{N}_{, i} \Omega_{, j}-\frac{3}{2} \tilde{N} \gamma^{i j} \Omega_{, i} \Omega, j+\frac{1}{6} \tilde{N} K^{2}-\frac{1}{4} \tilde{N} \Omega^{2} \tilde{R} \\
& +\frac{5}{4} \tilde{N} \Omega^{2} \mu_{\gamma}^{-2} \gamma_{i k} \gamma_{j l} \pi^{\operatorname{tr} i j} \pi^{\operatorname{tr} k l}+\frac{1}{2} \kappa \tilde{N} \Omega^{4}(\tilde{S}+2 \tilde{\rho})=0,
\end{aligned}
$$

where $\tilde{S} \equiv \gamma^{i j} \tilde{S}_{i j}$.

We also need to specify the spatial coordinates. In [3] we imposed a spatially harmonic gauge condition, which yielded an elliptic system for the shift (equation (2.15) in [3]). However, other choices are possible. For example, in [5] and in section

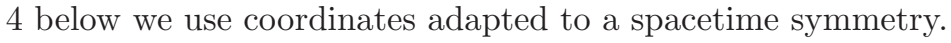

There is a residual conformal gauge freedom inherent in the decomposition (11). In 3 we fixed this by requiring the conformal scalar curvature $\tilde{R}$ to be constant; this resulted in an elliptic equation for $\Gamma=-2 \tilde{N} C$ (equation (2.12) in [3]). In section 4 the spherically symmetric conformal metric will be taken to be flat; this eliminates the conformal gauge freedom. 


\subsection{Regularity at future null infinity}

The evolution equation for the traceless momentum (18) and the elliptic equations (20) - 222) contain inverse powers of the conformal factor that are singular at $\mathscr{I}^{+}$. However in 3 we showed, for the vacuum case, that the formally singular terms in (18) can in fact be evaluated at $\mathscr{I}^{+}$in terms of regular conformal quantities, provided the constraints are satisfied and $\mathscr{I}^{+}$is shear free (see also [29). We also showed that these regularity conditions are preserved under the time evolution.

Our analysis exploited the fact that the elliptic equations (20)-(22) are degenerate at $\mathscr{I}^{+}$. This allows one to evaluate the first few radial derivatives of the fields explicitly at $\mathscr{I}^{+}$, where radius $r$ refers to a coordinate on a given spatial slice that is constant on the cut of the slice with $\mathscr{I}^{+}$. In particular, we obtained expressions for the first three radial derivatives of $\Omega$, the first two radial derivatives of $\tilde{N}$ and the first radial derivatives of $\pi^{\operatorname{tr} r i}$ at $\mathscr{I}^{+}$. This information was sufficient in order to evaluate the formally singular terms in (18) by applying L'Hospital's rule.

It is easy to see that the addition of matter does not affect those results, essentially because the matter terms in (20)-(22) are multiplied by sufficiently high powers of $\Omega$ that vanish at $\mathscr{I}^{+}$even after a certain number of derivatives are taken. More specifically, the third radial derivative of $\Omega$ at $\mathscr{I}^{+}$was obtained in 3 by evaluating the Laplacian of (20). Now the Laplacian of the matter contribution to (20) is $\mathcal{O}\left(\Omega^{2}\right)$ and hence does not contribute at $\mathscr{I}^{+}$. Clearly, the matter contributions to lower derivatives of $\Omega$ are multiplied by even higher powers of $\Omega$ and vanish at $\mathscr{I}^{+}$as well. The first radial derivative of $\pi^{\operatorname{tr} r i}$ was obtained by first multiplying (21) by $\Omega^{3}$ and then taking a radial derivative. After this operation the matter contribution is $\mathcal{O}\left(\Omega^{2}\right)$ and hence, again, does not contribute at $\mathscr{I}^{+}$. We found the second radial derivative

of $\tilde{N}$ by forming the linear combination $\Omega^{-1} \times(\underline{22})+\frac{1}{4} \Omega^{-1} \tilde{N} \times(20)$ and then taking a radial derivative. The matter contribution to the resulting expression is $\mathcal{O}\left(\Omega^{2}\right)$ and hence does not contribute at $\mathscr{I}^{+}$. Thus all the expressions we derived in order to evaluate the formally singular terms in (18) at $\mathscr{I}^{+}$are unchanged by the addition of matter. The matter term in (18) itself is $\mathcal{O}\left(\Omega^{2}\right)$ and thus vanishes at $\mathscr{I}^{+}$.

\section{Examples of matter models}

In this section we consider two matter models in detail, a conformally coupled scalar field and Yang-Mills theory. We write the matter equation of motion in $3+1$ form and work out the projections of the energy-momentum tensor that appear as source terms in the $3+1$ form of the Einstein equations.

\subsection{The conformally coupled scalar field}

The action for the Einstein-scalar field model with conformal coupling is given by

$$
S=\int\left(\frac{1}{2 \kappa}^{(4)} R-\frac{1}{2}^{(4)} g^{\mu \nu} \phi_{, \mu} \phi_{, \nu}-\frac{1}{12}{ }^{(4)} R \phi^{2}\right) \mu_{(4)} g \mathrm{~d}^{4} x .
$$

This differs from minimal coupling by the additional last term containing the fourdimensional Ricci scalar. As we shall see shortly, the advantage of the conformally coupled model is that it yields a conformally invariant evolution equation for the scalar field. We note however that the conformally coupled Einstein-scalar field equations are equivalent to the minimally coupled ones in the following sense [30, 10]: Suppose 
$\left(\phi^{\mathrm{cc}},{ }^{(4)} g_{\mu \nu}^{\mathrm{cc}}\right)$ is a solution to the conformally coupled equations. Then $\left(\phi^{\mathrm{mc}},{ }^{(4)} g_{\mu \nu}^{\mathrm{mc}}\right)$ is a solution to the minimally coupled equations, where

$$
\phi^{\mathrm{mc}}=\sqrt{6} \operatorname{arctanh}\left(\frac{1}{2} \phi^{\mathrm{cc}}\right), \quad{ }^{(4)} g_{\mu \nu}^{\mathrm{mc}}=\left[1-\frac{1}{4}\left(\phi^{\mathrm{cc}}\right)^{2}\right]^{(4)} g_{\mu \nu}^{\mathrm{cc}} .
$$

Varying (23) w.r.t. $\phi$ yields the equation of motion

$$
\square \phi-\frac{1}{6}{ }^{(4)} R \phi=0 \text {. }
$$

Defining a conformally rescaled field $\tilde{\phi} \equiv \Omega^{-1} \phi$ and using the transformation of the Ricci scalar A.4 , we find

$$
\tilde{\square} \tilde{\phi}-\frac{1}{6}{ }^{(4)} \tilde{R} \tilde{\phi}=\Omega^{-3}\left(\square \phi-\frac{1}{6}^{(4)} R \phi\right)=0 .
$$

The left-hand side of this equation is manifestly regular at $\mathscr{I}^{+}$.

Varying (23) w.r.t. ${ }^{(4)} g_{\mu \nu}$ produces Einstein's equations $G_{\mu \nu}=\kappa T_{\mu \nu}$ with energymomentum tensor

$T_{\mu \nu}=\phi_{, \mu} \phi_{, \nu}-\frac{1}{2} \phi^{(4)} \nabla_{\mu}{ }^{(4)} \nabla_{\nu} \phi+\frac{1}{4} \phi^{2(4)} R_{\mu \nu}-\frac{1}{4}{ }^{(4)} g_{\mu \nu}\left({ }^{(4)} g^{\rho \sigma} \phi_{, \rho} \phi_{, \sigma}+\frac{1}{6} \phi^{2(4)} R\right)$,

where an overall factor of $\frac{2}{3}$ has been absorbed into the definition of $\phi$, and the equation of motion (25) has been used to eliminate a $\square \phi$ term. This can also be written as $T_{\mu \nu}=\Omega^{2} \tilde{T}_{\mu \nu}$, where the conformally rescaled energy-momentum tensor has exactly the same form,

$\tilde{T}_{\mu \nu}=\tilde{\phi}_{, \mu} \tilde{\phi}_{, \nu}-\frac{1}{2} \tilde{\phi}^{(4)} \tilde{\nabla}_{\mu}{ }^{(4)} \tilde{\nabla}_{\nu} \tilde{\phi}+\frac{1}{4} \tilde{\phi}^{2(4)} \tilde{R}_{\mu \nu}-\frac{1}{4}{ }^{(4)} \gamma_{\mu \nu}\left({ }^{(4)} \gamma^{\rho \sigma} \tilde{\phi}_{, \rho} \tilde{\phi}_{, \sigma}+\frac{1}{6} \tilde{\phi}^{2}{ }^{(4)} \tilde{R}\right)$.

Using the $3+1$ decomposition of the scalar field Hessian (Appendix A.3) and the conformal spacetime Ricci tensor Appendix A.4), the equation of motion (26) can be written as

$$
\begin{aligned}
0=-\mathcal{L}_{\tilde{n}}^{2} \tilde{\phi}+\tilde{\nabla}_{i} \tilde{\nabla}^{i} \tilde{\phi}+C \mathcal{L}_{\tilde{n}} \tilde{\phi}+\tilde{N}^{-1} \gamma^{i j} \tilde{N}_{, i} \tilde{\phi}_{, j} \\
\quad-\frac{1}{6} \tilde{\phi}\left(-2 \tilde{N}^{-1} \tilde{\nabla}^{i} \tilde{\nabla}_{i} \tilde{N}+\tilde{R}+\frac{4}{3} C^{2}+\mu_{\gamma}^{-2} \gamma_{i k} \gamma_{j l} \pi^{\operatorname{tr} i j} \pi^{\operatorname{tr} k l}-2 \mathcal{L}_{\tilde{n}} C\right)
\end{aligned}
$$

(Recall that we are raising and lowering spatial indices with $\gamma_{i j}$.) In general, the time derivative of the conformal mean curvature $C$ cannot be evaluated explicitly during an evolution, e.g. if $\Gamma=-2 \tilde{N} C$ is determined by solving an elliptic equation as in 3 . This problem can be avoided by introducing a new variable $\tilde{\psi}$ and writing (29) as a first-order (in time) system

$$
\begin{aligned}
& \mathcal{L}_{\tilde{n}} \tilde{\phi}=\tilde{\psi}+\frac{1}{3} C \tilde{\phi}, \\
& \mathcal{L}_{\tilde{n}} \tilde{\psi}=\tilde{\nabla}_{i} \tilde{\nabla}^{i} \tilde{\phi}+\frac{2}{3} C \tilde{\psi}+\tilde{N}^{-1} \gamma^{i j} \tilde{N}_{, i} \tilde{\phi}_{, j} \\
& \quad-\frac{1}{6} \tilde{\phi}\left(-2 \tilde{N}^{-1} \tilde{\nabla}^{i} \tilde{\nabla}_{i} \tilde{N}+\tilde{R}+\mu_{\gamma}^{-2} \gamma_{i k} \gamma_{j l} \pi^{\operatorname{tr} i j} \pi^{\operatorname{tr} k l}\right) .
\end{aligned}
$$

The matter source terms in the geometry equations evaluate to

$$
\begin{aligned}
& \tilde{\rho}=-\frac{1}{2} \tilde{\phi} \tilde{\nabla}_{i} \tilde{\nabla}^{i} \tilde{\phi}+\frac{1}{4} \tilde{\phi}_{, i} \tilde{\phi}^{, i}+\frac{3}{4} \tilde{\psi}^{2}+\frac{1}{8} \tilde{\phi}^{2} \tilde{R}-\frac{1}{8} \tilde{\phi}^{2} \mu_{\gamma}^{-2} \gamma_{i k} \gamma_{j l} \pi^{\operatorname{tr} i j} \pi^{\operatorname{tr} k l}, \\
& \tilde{J}^{i}=\gamma^{i j}\left(-\tilde{\phi}_{, j} \tilde{\psi}+\frac{1}{2} \tilde{\phi} \tilde{\psi}_{, j}\right)-\frac{1}{4} \mu_{\gamma}^{-1} \tilde{\nabla}_{j}\left(\tilde{\phi}^{2} \pi^{\operatorname{tr} i j}\right), \\
& \tilde{S}=\tilde{\rho}, \\
& \tilde{S}^{\operatorname{tr} i j}=-\frac{1}{2} \tilde{\phi}\left[\tilde{\nabla}^{i} \tilde{\nabla}^{j} \tilde{\phi}\right]^{\operatorname{tr}}+\left[\tilde{\phi}^{, i} \tilde{\phi}^{, j}\right]^{\operatorname{tr}}+\frac{1}{2} \tilde{\phi} \tilde{\psi} \mu_{\gamma}^{-1} \pi^{\operatorname{tr} i j} \\
& \quad+\frac{1}{4} \tilde{\phi}^{2}\left(\mu_{\gamma}^{-1} \mathcal{L}_{\tilde{n}} \pi^{\operatorname{tr} i j}+2 \mu_{\gamma}^{-2} \gamma_{k l} \pi^{\operatorname{tr} i k} \pi^{\operatorname{tr} j l}-\tilde{N}^{-1}\left[\tilde{\nabla}^{i} \tilde{\nabla}^{j} \tilde{N}\right]^{\operatorname{tr}}+\tilde{R}^{\operatorname{tr} i j}\right) .
\end{aligned}
$$

The equation of motion (29) has been used in order to eliminate a $\mathcal{L}_{\tilde{n}}^{2} \tilde{\phi}$ term in (32). Note that (35) contains $\mathcal{L}_{\tilde{n}} \pi^{\text {trij. }}$. When (35) is substituted in the evolution equation (18) for the traceless momentum, the resulting equation must be solved for $\mathcal{L}_{\tilde{n}} \pi^{\operatorname{tr} i j}$. 


\subsection{Yang-Mills theory}

The fundamental field of Yang-Mills theory is a connection or vector potential $A_{\mu}^{(a)}$ which in addition to the spacetime index $\mu$ carries an index $(a)$ referring to the internal gauge group. We use internal indices from the beginning of the Latin alphabet $a, b, \ldots$ ranging over $1,2, \ldots, N$, where $N$ is the dimension of the associated Lie algebra, e.g. $N=3$ if the gauge group is $S U(2)$. Repeated internal indices are summed over.

Since the Yang-Mills equations are conformally invariant, we choose to work directly in the conformal spacetime here. The Yang-Mills connection and its conformal counterpart are identical, $A_{\mu}^{(a)}=\tilde{A}_{\mu}^{(a)}$. We regard

$$
\tilde{A}_{0}^{(a)} \equiv-\tilde{\psi}^{(a)}
$$

as a gauge variable that can be freely chosen.

The Yang-Mills field strength tensor is defined as

$$
\tilde{F}_{\mu \nu}^{(a)}=\partial_{\mu} \tilde{A}_{\nu}^{(a)}-\partial_{\nu} \tilde{A}_{\mu}^{(a)}+f^{a b c} \tilde{A}_{\mu}^{(b)} \tilde{A}_{\nu}^{(c)},
$$

where the symbol $f^{a b c}$ is totally antisymmetric in the chosen basis of the Lie algebra. For $S U(2)$ we may write $f^{a b c}=g[a b c]$, where $g$ is a constant; we set $g=-2$ for our numerical evolutions in section [6. The symbol $[a b c]$ is totally antisymmetric with $[123]=1$.

We introduce the electric field

$$
\tilde{\mathcal{D}}^{i(a)} \equiv \sqrt{-{ }^{(4)} \gamma} \tilde{F}^{0 i(a)}=\tilde{N} \mu_{\gamma} \tilde{F}^{0 i(a)}
$$

and magnetic field

$$
\tilde{\mathcal{B}}^{i(a)}=\frac{1}{2} \mu_{\gamma} \epsilon^{i j k} \tilde{B}_{j k}^{(a)}=\frac{1}{2}[i j k] \tilde{F}_{j k}^{(a)},
$$

where

$$
\tilde{B}_{i j}^{(a)} \equiv \tilde{F}_{i j}^{(a)}
$$

denotes the spatial part of the field strength tensor and $\epsilon_{i j k}$ is the alternating symbol associated with the conformal spatial metric $\gamma_{i j}$. Note that $\tilde{\mathcal{D}}^{i}$ and $\tilde{\mathcal{B}}^{i}$ are vector densities of weight one.

The energy-momentum tensor is given by

$$
\tilde{T}_{\mu \nu}=\tilde{F}_{\mu \rho}^{(a)} \tilde{F}_{\nu}{ }^{\rho(a)}-\frac{1}{4}{ }^{(4)} \gamma_{\mu \nu} \tilde{F}_{\rho \sigma}^{(a)} \tilde{F}^{\rho \sigma(a)} .
$$

The projections defined in (13) take the form

$$
\begin{aligned}
& \tilde{\rho}=\frac{1}{2} \mu_{\gamma}^{-2}\left(\tilde{\mathcal{D}}^{i(a)} \tilde{\mathcal{D}}_{i}^{(a)}+\tilde{\mathcal{B}}^{i(a)} \tilde{\mathcal{B}}_{i}^{(a)}\right), \\
& \tilde{J}_{i}=\mu_{\gamma}^{-1}[i j k] \tilde{\mathcal{D}}^{j(a)} \tilde{\mathcal{B}}^{k(a)}, \\
& \tilde{S}=\tilde{\rho}, \\
& \tilde{S}^{\operatorname{tr} i j}=-\mu_{\gamma}^{-2}\left[\tilde{\mathcal{D}}^{i(a)} \tilde{\mathcal{D}}^{j(a)}+\tilde{\mathcal{B}}^{i(a)} \tilde{\mathcal{B}}^{j(a)}\right]^{\operatorname{tr}} .
\end{aligned}
$$

Energy-momentum conservation yields the Yang-Mills field equations

$$
{ }^{(4)} \tilde{\nabla}_{\mu} \tilde{F}^{\mu \nu(a)}+f^{a b c} \tilde{A}_{\mu}^{(b)} \tilde{F}^{\mu \nu(c)}=0 .
$$

Contracting this with $\tilde{n}_{\nu}$, we obtain the constraint

$$
\partial_{i} \tilde{\mathcal{D}}^{i(a)}+f^{a b c} A_{i}^{(b)} \tilde{\mathcal{D}}^{i(c)}=0 ;
$$


contracting with $\gamma_{\nu}{ }^{i}$ instead,

$$
\begin{aligned}
& \partial_{t} \tilde{\mathcal{D}}^{i(a)}-\left(X^{j} \tilde{\mathcal{D}}^{i(a)}\right)_{, j}+X^{i}{ }_{, j} \tilde{\mathcal{D}}^{j(a)}= \\
& \partial_{j}\left(\tilde{N} \mu_{\gamma} \tilde{B}^{i j(a)}\right)+\tilde{N} \mu_{\gamma} f^{a b c} \tilde{A}_{j}^{(b)} \tilde{B}^{i j(c)}+f^{a b c}\left(\psi^{(b)}+X^{k} A_{k}^{(b)}\right) \tilde{\mathcal{D}}^{i(c)}
\end{aligned}
$$

An evolution equation for the vector potential is obtained from the definition of $\tilde{F}_{0 i}^{(a)}$,

$$
\partial_{t} \tilde{A}_{i}^{(a)}=-\tilde{N} \mu_{\gamma}^{-1} \tilde{\mathcal{D}}_{i}^{(a)}-X^{j} \tilde{B}_{i j}^{(a)}-\tilde{\psi}_{, i}^{(a)}-f^{a b c} \tilde{A}_{i}^{(b)} \tilde{\psi}^{(c)} .
$$

Equations (49) and (48) constitute the evolution equations for the independent variables $\tilde{A}_{i}^{(a)}$ and $\tilde{\mathcal{D}}^{i(a)}$. Note that $\tilde{B}_{i j}^{(a)}$ is to be expressed in terms of $\tilde{A}_{i}^{(a)}$ and its spatial derivatives using definitions (37) and (40).

\section{Reduction to spherical symmetry}

In this section we reduce the formulation of the Einstein equations presented in section 2.2 and the specific matter models of section 3 to spherical symmetry. Special care is needed in the case of Yang-Mills theory.

\subsection{Einstein equations in isotropic gauge}

We work in spherical polar coordinates $t, r, \theta, \varphi$. All fields are functions of $t$ and $r$ only. Partial derivatives w.r.t. time and radius are denoted by ${ }^{\circ}=\partial_{t}$ and ${ }^{\prime}=\partial_{r}$.

In spherical symmetry we may take the spatial conformal metric to be flat,

$$
\gamma_{i j}=\operatorname{diag}\left(1, r^{2}, r^{2} \sin ^{2} \theta\right) \text {. }
$$

The shift vector has a radial component only, and we set $X^{r} \equiv r X$. The traceless momentum is diagonal and has only one independent component,

$$
\pi^{\operatorname{tr} r r}=-2 r^{2} \pi^{\operatorname{tr} \theta \theta}=-2 r^{2} \sin ^{2} \theta \pi^{\operatorname{tr} \varphi \varphi} \equiv r^{2} \sin \theta \hat{\pi} .
$$

(We have pulled out a factor of $\mu_{\gamma}=r^{2} \sin \theta$ in the definition of the variable $\hat{\pi}$ in order to avoid frequent divisions by $\mu_{\gamma}$ in the final equations.)

Preservation of (50) under the evolution equation (17) implies the spatial gauge condition

$$
r X^{\prime}=-\frac{3}{2} \tilde{N} \hat{\pi} .
$$

This implies that $\hat{\pi}$ is actually $\mathcal{O}\left(r^{2}\right)$ near the origin and so we set

$$
\hat{\pi} \equiv r^{2} \pi \text {. }
$$

We also note the expression for the conformal mean curvature in our gauge,

$$
C=\tilde{N}^{-1} \tilde{\nabla}_{i} X^{i}=-\frac{3}{2} r^{2} \pi+3 \tilde{N}^{-1} X .
$$

The reduction of the Einstein equations is as follows. The Hamiltonian constraint (20) reads

$$
-4 \Omega \Omega^{\prime \prime}+6 \Omega^{\prime 2}-8 \Omega r^{-1} \Omega^{\prime}+\frac{3}{2} \Omega^{2} r^{4} \pi^{2}-\frac{2}{3} K^{2}+2 \kappa \Omega^{4} \tilde{\rho}=0 .
$$

The momentum constraint (21) is

$$
\Omega\left(r \pi^{\prime}+5 \pi\right)-2 r \Omega^{\prime} \pi+\kappa \Omega^{3} r^{-1} \tilde{J}^{r}=0 .
$$

The CMC slicing condition (22) is

$$
\begin{gathered}
-\Omega^{2} \tilde{N}^{\prime \prime}+3 \Omega \Omega^{\prime} \tilde{N}^{\prime}-2 \Omega^{2} r^{-1} \tilde{N}^{\prime}-\frac{3}{2} \Omega^{\prime 2} \tilde{N}+\frac{1}{6} \tilde{N} K^{2}+\frac{15}{8} \tilde{N} \Omega^{2} r^{4} \pi^{2} \\
+\frac{1}{2} \kappa \tilde{N} \Omega^{4}(\tilde{S}+2 \tilde{\rho})=0 .
\end{gathered}
$$


The evolution equation for the conformal factor (16) is

$$
\dot{\Omega}=r X \Omega^{\prime}-X \Omega+\tilde{N}\left(\frac{1}{2} \Omega r^{2} \pi-\frac{1}{3} K\right)
$$

and that for the traceless momentum (18)

$$
\begin{aligned}
\dot{\pi}=r X \pi^{\prime}+3 X & +\frac{2}{3} r^{-1}\left(r^{-1} \tilde{N}^{\prime}\right)^{\prime} \\
& +\tilde{N}\left[-\frac{4}{3} \Omega^{-1} r^{-1}\left(r^{-1} \Omega^{\prime}\right)^{\prime}-\frac{2}{3} \Omega^{-1} K \pi-\frac{1}{2} r^{2} \pi^{2}+\kappa \Omega^{2} r^{-2} \tilde{S^{t r} r}\right] .
\end{aligned}
$$

4.1.1. Regularity at the origin. The fields $\Omega, \pi, \tilde{N}$ and $X$ are even functions of $r$. It follows from the general behaviour near the origin of spherically symmetric tensor fields [31, 32] that the $r$-component of a vector $\tilde{J}^{i}$ is $\mathcal{O}(r)$ and the $r r$-component of a tracefree symmetric tensor $\tilde{S}^{\operatorname{tr} i j}$ is $\mathcal{O}\left(r^{2}\right)$ near the origin; this can also be seen explicitly for the specific matter models in the following subsections. Hence the terms $\rho, r^{-1} \tilde{J}^{r}, \tilde{S}$ and $r^{-2} \tilde{S}^{\operatorname{tr} r r}$ appearing as sources in (55)-(59) are also regular even functions of $r$. Keeping this in mind, equations (55)-(59) have been written in a form that is manifestly regular at the origin.

4.1.2. Regularity at future null infinity. For completeness and for later use we also state the results of our regularity analysis at $\mathscr{I}^{+}$here. In the following $\hat{=}$ denotes equality at $\mathscr{I}^{+}$. By definition,

$$
\Omega \hat{=} 0 \text {. }
$$

The Hamiltonian constraint (55) and momentum constraint (56) yield

$$
\begin{aligned}
& \Omega^{\prime} \hat{=} r \Omega^{\prime \prime} \hat{=}-\frac{1}{3} K, \quad \Omega^{\prime \prime \prime} \hat{=} 0 \\
& \pi \hat{=} \pi^{\prime} \hat{=} 0
\end{aligned}
$$

From the CMC condition (57) we obtain

$$
r \tilde{N}^{\prime} \hat{=} r^{2} \tilde{N}^{\prime \prime} \hat{=} \tilde{N}
$$

Note that the value of $\tilde{N}$ at $\mathscr{I}^{+}$can be freely chosen; see section 5.2 for our particular choice. Preservation of (60) under the evolution equation (58) implies

$$
r X \hat{=}-\tilde{N} \text {. }
$$

The isotropic gauge condition (52) further yields

$$
X^{\prime} \hat{=} X^{\prime \prime} \hat{=} 0
$$

Given these results it can be verified using L'Hospital's rule that the evolution equation (59) for the traceless momentum reduces to

$$
\partial_{t} \pi \hat{=} 0
$$

consistent with (62). 
4.2. The conformally coupled scalar field

The scalar field evolution equations (30)-(31) take the form

$\dot{\tilde{\phi}}=r X \tilde{\phi}^{\prime}+X \tilde{\phi}+\tilde{N}\left(-\frac{1}{2} \tilde{\phi} r^{2} \pi+\tilde{\psi}\right)$,

$\dot{\tilde{\psi}}=\left(r X \tilde{\psi}+\tilde{N} \tilde{\phi}^{\prime}\right)^{\prime}+X \tilde{\psi}+\tilde{N}\left(2 r^{-1} \tilde{\phi}^{\prime}-\frac{1}{4} \tilde{\phi} r^{4} \pi^{2}+\frac{1}{2} \tilde{\psi} r^{2} \pi\right)+\frac{1}{3} \tilde{\phi}\left(\tilde{N}^{\prime \prime}+2 r^{-1} \tilde{N}^{\prime}\right)$.

The source terms (32)-(35) appearing in the Einstein equations are

$\tilde{\rho}=-\frac{1}{2} \tilde{\phi}\left(\tilde{\phi}^{\prime \prime}+2 r^{-1} \tilde{\phi}^{\prime}\right)+\frac{1}{4} \tilde{\phi}^{\prime 2}+\frac{3}{4} \tilde{\psi}^{2}-\frac{3}{16} \tilde{\phi}^{2} r^{4} \pi^{2}$,

$r^{-1} \tilde{J}^{r}=-r^{-1} \tilde{\phi}^{\prime} \tilde{\psi}+\frac{1}{2} \tilde{\phi} r^{-1} \tilde{\psi}^{\prime}-\frac{1}{2} \tilde{\phi} \tilde{\phi}^{\prime} r \pi-\frac{1}{4} \tilde{\phi}^{2}\left(r \pi^{\prime}+5 \pi\right)$,

$\tilde{S}=\tilde{\rho}$,

$r^{-2} \tilde{S}^{\operatorname{tr} r r}=-\frac{1}{3} \tilde{\phi} r^{-1}\left(r^{-1} \tilde{\phi}^{\prime}\right)^{\prime}+\frac{2}{3}\left(r^{-1} \tilde{\phi}^{\prime}\right)^{2}+\frac{1}{2} \tilde{\phi} \tilde{\psi} \pi$

$$
+\frac{1}{4} \tilde{\phi}^{2}\left[\tilde{N}^{-1}\left(\dot{\pi}-r X \pi^{\prime}-3 X \pi\right)+\frac{1}{2} r^{2} \pi^{2}-\frac{2}{3} \tilde{N}^{-1} r^{-1}\left(r^{-1} \tilde{N}^{\prime}\right)^{\prime}\right] .
$$

Notice again that these equations are manifestly regular in the origin, given that $\tilde{\phi}$ and $\tilde{\psi}$ are even functions of $r$.

\subsection{Yang-Mills theory}

In the case of Yang-Mills it is more convenient to work in Cartesian coordinates, so indices $i, j, \ldots$ will refer to Cartesian coordinates in this subsection. Hence the conformal metric is now $\gamma_{i j}=\delta_{i j}$, with $\mu_{\gamma}=1$, and the shift vector is $X^{i}=X x^{i}$.

We take the gauge group to be $S U(2)$. The most general ansatz for the spherically symmetric Yang-Mills connection is of the form [33, 34, 35]

$$
\tilde{A}^{i(a)}=[a i j] x^{j} F+\left(x^{a} x^{i}-r^{2} \delta^{a i}\right) H+\delta^{a i} L, \quad \tilde{A}_{0}^{(a)}=G x^{a} .
$$

Here $F, H, L$ and $G$ are functions of $t$ and $r$ only.

Similarly, we write the electric field as

$$
\tilde{\mathcal{D}}^{i(a)}=[a i j] x^{j} D_{F}+\left(x^{a} x^{i}-r^{2} \delta^{a i}\right) D_{H}+\delta^{a i} D_{L} .
$$

The evolution equations for the vector potential (49) read

$$
\begin{aligned}
& \dot{F}=r X F^{\prime}-\tilde{N} D_{F}+2 X F+g(G-X L)\left(L-r^{2} H\right), \\
& \dot{H}=r X H^{\prime}-\tilde{N} D_{H}+r^{-1} G^{\prime}+g(G-X L) F+X\left(3 H-r^{-1} L^{\prime}\right), \\
& \dot{L}=-\tilde{N} D_{L}+r G^{\prime}+G .
\end{aligned}
$$

The evolution equations for the electric field (48) are

$$
\begin{aligned}
\dot{D}_{F}=\left(r X D_{F}-\right. & \left.\tilde{N} F^{\prime}\right)^{\prime}+2 X D_{F}+g\left(D_{L}-r^{2} D_{H}\right)(G-X L) \\
& +\tilde{N}\left[-4 r^{-1} F^{\prime}-2 g L r H^{\prime}+g r^{-1} L^{\prime}\left(3 L-r^{2} H\right)\right] \\
& +r^{-1} \tilde{N}^{\prime}\left[-2 F+g L\left(L-r^{2} H\right)\right]+\tilde{N} g\left[-3 F^{2}-r^{2} H^{2}-4 H L\right. \\
& \left.\quad+g\left(r^{2} F^{3}-2 r^{2} F H L+r^{4} F H^{2}+2 F L^{2}\right)\right], \\
\dot{D}_{H}=\left(r X D_{H}-\right. & \left.\tilde{N} H^{\prime}\right)^{\prime}-r^{-1}\left(X D_{L}-\tilde{N} r^{-1} L^{\prime}\right)^{\prime}+r^{-1} \tilde{N}^{\prime}(-3 H+g F L) \\
& +g D_{F}\left(G+X L-2 X r^{2} H\right)+X D_{H}\left(1+2 g r^{2} F\right)-2 g X F D_{L} \\
& +\tilde{N}\left[-4 r^{-1} H^{\prime}+g\left(2 H r F^{\prime}-2 F r H^{\prime}+3 F r^{-1} L^{\prime}\right)\right] \\
& +\tilde{N} g\left\{-4 F H+g\left[F^{2}\left(L+r^{2} H\right)+r^{4} H^{3}-r^{2} H^{2} L\right]\right\}, \\
\dot{D}_{L}=X[2(1- & \left.\left.g r^{2} F\right)\left(D_{L}-r^{2} D_{H}\right)+2 g r^{2} D_{F}\left(L-r^{2} H\right)\right] \\
& +2 \tilde{N}\left(1-g r^{2} F\right)\left(3 H+r H^{\prime}-r^{-1} L^{\prime}-g F L\right) \\
& -2 \tilde{N} g\left(L-r^{2} H\right)\left[r F^{\prime}-g r^{2} H\left(L-r^{2} H\right)+2 F-g\left(L-r^{2} H\right)^{2}\right] .
\end{aligned}
$$


The Yang-Mills constraint (47) is

$$
r^{-1} D_{L}^{\prime}+2 g F\left(D_{L}-r^{2} D_{H}\right)+2 D_{H}+2 g\left(r^{2} H-L\right) D_{F}=0 .
$$

The components of the magnetic field

$$
\tilde{\mathcal{B}}^{i(a)}=[a i j] x^{j} B_{F}+\left(x^{a} x^{i}-r^{2} \delta^{a i}\right) B_{H}+\delta^{a i} B_{L}
$$

evaluate to

$$
\begin{aligned}
& B_{F}=-3 H-r H^{\prime}+r^{-1} L^{\prime}+g F L, \\
& B_{H}=r^{-1} F^{\prime}-g H\left(L-r^{2} H\right)+g F^{2}, \\
& B_{L}=-2 F+g\left(L-r^{2} H\right)^{2}+g r^{2} F^{2} .
\end{aligned}
$$

In terms of these, the matter sources in the Einstein equations are given by

$$
\begin{aligned}
& \tilde{\rho}=\tilde{S}=\frac{1}{2}\left[3 D_{L}^{2}-2 r^{2}\left(2 D_{L} D_{H}-D_{F}^{2}-r^{2} D_{H}^{2}\right)\right. \\
& \left.+3 B_{L}^{2}-2 r^{2}\left(2 B_{L} B_{H}-B_{F}^{2}-r^{2} B_{H}^{2}\right)\right], \\
& r^{-1} \tilde{J}^{r}=2\left[D_{L} B_{F}-D_{F} B_{L}+r^{2}\left(D_{F} B_{H}-D_{H} B_{F}\right)\right], \\
& \tilde{r}^{-2} \tilde{S}^{\operatorname{tr} r}=-\frac{2}{3}\left(2 D_{L} D_{H}-D_{F}^{2}-r^{2} D_{H}^{2}+2 B_{L} B_{H}-B_{F}^{2}-r^{2} B_{H}^{2}\right) .
\end{aligned}
$$

We are free to impose one gauge condition on the Yang-Mills connection, and we choose radial gauge $L=0$. The evolution equation (77) for $L$ now turns into an ODE determining $G$,

$$
r G^{\prime}+G-\tilde{N} D_{L}=0 .
$$

If the initial data are such that $H=D_{H}=0$ then $D_{L}=0$ is a solution to (81), and $G=0$ (i.e. temporal gauge) is a solution to (89). (There are other solutions to these last two equations but the boundary conditions we impose will single out the given ones.) These conditions are preserved under the time evolution, i.e. $H=G=D_{H}=D_{L}=0$ at all times. This reduced system is sometimes referred to as purely magnetic or as the gravitational sector of the Einstein-Yang-Mills equations, whereas the remaining system is called the sphaleron sector [36. While

the gravitational sector has been widely studied numerically (e.g. in [37, 36, 23), we are not aware of any numerical simulations using the full system.

We also note that the ansatz (73) is often written (e.g. in [36) in a different gauge known as Abelian gauge,

$$
\tilde{A}=w \tau^{\theta} \mathrm{d} \theta+\left(\cot \theta \tau^{r}+w \tau^{\phi}\right) \sin \theta \mathrm{d} \theta
$$

where $\tau^{i}$ denote the generators of $S U(2)$. The two ansätze are related by a (singular) $S U(2)$ gauge transformation (see e.g. [38]).

\section{Numerical method}

In this section we describe the numerical methods we use in order to solve the spherically symmetric reduction of the Einstein equations on hyperboloidal slices derived in section 4 We begin by summarising our evolution scheme. Particular care is spent on the boundary conditions. We then describe the finite-difference discretisation, time integration method and elliptic solver. Finally we explain how apparent horizons are detected and excised. The code has been implemented in Python. 


\subsection{Evolution scheme}

Our fundamental geometric variables are $\Omega, \pi, \tilde{N}$ and $X$. The matter variables are either $\tilde{\phi}$ and $\tilde{\psi}$ for the scalar field, or $F, H, G, D_{F}, D_{H}$ and $D_{L}$ for Yang-Mills.

The numerical evolution proceeds as follows. At each time step, we solve

(i) the Yang-Mills constraint (81) for $D_{L}$,

(ii) the Einstein constraints (55) and (56) for $\Omega$ and $\pi$. If the source $\tilde{J}^{r}$ of the momentum constraint is independent of $\pi$, as is the case for Yang-Mills, then the two equations may be decoupled by setting $\pi=\Omega^{2} P$, which turns the momentum constraint (56) into

$$
r P^{\prime}+5 P+\kappa r^{-1} \tilde{J}^{r}=0 .
$$

This is solved first and the solution for $P$ is then substituted in the Hamiltonian constraint (55). For the conformally coupled scalar field, the two constraints cannot be decoupled in this way because $\tilde{J}^{r}$ depends on $\pi$ (cf. equation (70)). In this case the Einstein constraints must be solved as a coupled system.

(iii) the slicing condition (57) for $\tilde{N}$,

(iv) the isotropic spatial gauge condition (52) for $X$,

(v) the Yang-Mills radial gauge condition (89) for $G$.

(Obviously steps (i) and (v) are only included for Yang-Mills matter.) The matter variables are then evolved to the next time step using either (67) and (66) for $\tilde{\phi}$ and $\tilde{\psi}$ or (75), (76), (78) and (79) for $F, H, D_{F}$ and $D_{H}$.

The evolution equations (58), (59) and (80) for $\Omega, \pi$ and $D_{L}$ are not solved explicitly but are evaluated during the numerical evolution as a consistency check.

\subsection{Boundary conditions}

We discuss the boundary conditions for each of the equations in turn. We need to distinguish between two cases, a regular centre and a black hole with an inner excision boundary. The outer boundary is always placed at $\mathscr{I}^{+}$.

The Yang-Mills constraint (81) is a first-order ODE for $D_{L}$ that requires one Dirichlet boundary condition. The value of $D_{L}$ at either the origin or the excision boundary is obtained by evolving $D_{L}$ there according to its evolution equation (80).

The Hamiltonian constraint (55) is a second-order ODE for $\Omega$, and we regard it as a two-point boundary value problem. For a regular centre, the inner boundary condition follows from the fact that $\Omega$ is an even function of $r: \Omega^{\prime}=0$ at $r=0$. For an excised black hole, we use the evolution equation (58) to find the value of $\Omega$ at the excision boundary. The outer boundary condition at $\mathscr{I}^{+}$is $\Omega \hat{=} 0$ in both cases.

The momentum constraint (56) is a first-order ODE for $\pi$. At an excision boundary we use the evolution equation (59) to obtain a Dirichlet boundary condition for $\pi$ there. For a regular centre, however, there is a unique solution to (56) that is regular at $r=0$. This can be seen immediately by evaluating (56) at $r=0$, where the equation implies a Dirichlet condition for $\pi$. Thus no additional boundary condition is imposed at $r=0$.

The CMC slicing condition (57) is a second-order ODE for $\tilde{N}$ that we regard as a two-point boundary value problem. At a regular origin $r=0$ the boundary condition is $\tilde{N}^{\prime}=0$. At an excision boundary we choose to freeze the value of $\tilde{N}$ from the time 
when the black hole is excised. At $\mathscr{I}^{+}$we choose the conformal lapse such that time $t$ corresponds to proper time $\tau$ of an observer at $\mathscr{I}^{+}$. This is given by

$$
-\mathrm{d} \tau^{2}=\Omega^{-2}\left(-\tilde{N}^{2}+r^{2} X^{2}\right) \mathrm{d} t^{2} .
$$

Using the results of our regularity analysis (section 4.1.2), the formally singular term on the right-hand side can be shown to have a regular limit at $\mathscr{I}^{+}$,

$$
\Omega^{-2}\left(\tilde{N}^{2}-r^{2} X^{2}\right) \hat{=} 9 r^{-2} K^{-2} \tilde{N}^{2} .
$$

Hence choosing $t$ to coincide with $\tau$ at $\mathscr{I}^{+}$corresponds to setting

$$
\tilde{N} \hat{=} \frac{1}{3} K r \text {. }
$$

The isotropic spatial gauge condition (52) is a first-order ODE for $X$. The boundary condition for $X$ at $\mathscr{I}^{+}$follows from preservation of $\Omega \hat{=} 0$ under the evolution equation (58), namely, $X \hat{=}-r^{-1} \tilde{N}$.

Finally we consider the Yang-Mills radial gauge condition (89). This first-order ODE for $G$ has a unique solution that is regular at $r=0$. (Evaluating the equation at $r=0$ implies a Dirichlet condition for $G$.) Thus no additional boundary condition must be imposed at $r=0$. For an inner excision boundary we choose to freeze the value of $G$ there from the time of excision.

Since the outer boundary at $\mathscr{I}^{+}$is null and the inner excision boundary is spacelike, the evolution equations (in particular those for the matter variables) do not require any boundary conditions there. Near a regular origin the evolution equations need not be modified either. The way we discretise the equations near the boundaries is described in the following subsection.

\subsection{Discretisation}

The numerical domain is an interval $r \in[0,1]$ for a regular centre or $\left[r_{\min }, 1\right]$ for an excised centre. In both cases the outer boundary at $r=1$ corresponds to $\mathscr{I}^{+}$.

For solutions containing a black hole, the fields typically have steep gradients close to the inner (excision) boundary and hence it is advisable to introduce a non-uniform grid. We do this by introducing a new radial coordinate $x$ with respect to which the numerical grid is uniform, combined with a map

$$
r:[0,1] \rightarrow\left[r_{\min }, 1\right], \quad x \mapsto r(x),
$$

that is steeper near $x=1$ than near $x=0$, thus providing more resolution close to the inner boundary. As in 5 we choose

$$
r(x)=Q_{1} x^{2}+\left(1-r_{\min }-Q_{1}\right) x+r_{\min },
$$

where $0 \leqslant Q_{1}<1$ is a constant, typically $Q_{1}=0.5$. For evolutions with a regular centre, we use $r(x)=x$ for the numerical results shown here. More generally, we have implemented a map

$$
r(x)=Q_{2} x+\left(1-Q_{2}\right) x^{3},
$$

with $0 \leqslant Q_{2} \leqslant 1$ a constant. Note that since $r(x)$ in (97) is an odd function of $x$, the parity of a grid function with respect to $x$ will be the same as with respect to $r$. (This is not the case for (96) but parity considerations are only relevant for regularity at the origin.)

The interval $x \in[0,1]$ is covered by equidistant grid points. For a regular centre we use a grid that is staggered about the origin,

$$
x_{i}=\left(i+\frac{1}{2}\right) h, \quad 0 \leqslant i \leqslant N,
$$


whereas for an excised centre we use an unstaggered grid

$$
x_{i}=i h, \quad 0 \leqslant i \leqslant N,
$$

so that $r_{0} \equiv r\left(x_{0}\right)=r(0)=r_{\text {min }}$ lies on the excision boundary. In both cases, the grid is chosen such that $r_{N} \equiv r\left(x_{N}\right)=1$.

Derivatives with respect to $x$ are discretised using fourth-order accurate finite differences. Explicit expressions for the finite-difference operators can be found in Appendix $\mathrm{C}$ of [5. We use one-sided differences near $\mathscr{I}^{+}$and the inner excision boundary. For a regular centre, the usual centred finite-difference operators are used near the origin by formally extending the staggered grid (98) to negative values of $i$ and using the fact that all the evolved variables are even functions of $r$ (and hence of $x$ ), i.e. replacing

$$
x_{-1}=x_{0}, \quad x_{-2}=x_{1}, \quad x_{-3}=x_{2}, \quad \ldots \quad .
$$

The reader will have noticed that we have written the highest derivatives in the matter evolution equations (68) and (78)-(79) in flux-conservative form. When discretising these equations, we apply the finite-difference operators in prescisely the order in which the terms in the continuum equations are written. This has been found to be essential for the stability of the method.

\subsection{Time integration}

A fourth-order Runge-Kutta method is used in order to integrate the evolution equations forward in time. At each full timestep, the elliptic equations are solved as described below in section [5.5. At the substeps of the Runge-Kutta algorithm, we have found it sufficient to extrapolate the solution to the elliptic equations from previous timesteps (using a cubic polynomial approximation) instead of solving the equations explicitly. Kreiss-Oliger dissipation [39] is added in order to ensure stability; see Appendix $\mathrm{C}$ of [5]. For a regular centre, the dissipation operator is evaluated up to the innermost grid point using the rule (100). No dissipation is added at the outermost two grid points near $\mathscr{I}^{+}$and the excision boundary.

\subsection{Elliptic solver}

The elliptic equations listed in section [5.1, which are in fact ODEs in spherical symmetry, are solved using a combination of a Newton-Raphson iteration and a direct band-diagonal solver. The Newton-Raphson iteration is actually only needed for the Hamiltonian constraint (55), all the other equations are linear. The matrices arising from our fourth-order finite-difference discretisation are pentadiagonal, except near $\mathscr{I}^{+}$and the excision boundary, where one-sided differences with wider stencils are used; a few Gaussian eliminations are applied by hand in order to reduce the matrix to pentadiagonal form there. We use the Python routine numpy.linalg. solve_banded (a straightforward generalisation of the Thomas algorithm) in order to solve this pentadiagonal linear system.

\subsection{Apparent horizon finder, black hole excision and Bondi mass}

We detect the formation of an apparent horizon by tracking the optical scalars $\theta_{ \pm}$ during the evolution. These are defined as follows. Let $n_{\mu}=\Omega^{-1} \tilde{N}(\mathrm{~d} t)_{\mu}$ denote the (physical) unit timelike normal to a given $t=$ const slice and $s^{\mu}=\Omega(\partial / \partial r)^{\mu}$ the unit 
outward normal to an $r=$ const surface within that slice. The outgoing and ingoing null normals to this surface are $l_{ \pm}^{\mu}=n^{\mu} \pm s^{\mu}$. The optical scalars or null expansions are now given by

$$
\theta_{ \pm}=(\ln R)_{, \mu} l_{ \pm}^{\mu}=\frac{1}{3} K-\frac{1}{2} \Omega r^{2} \pi \pm\left(r^{-1} \Omega-\Omega^{\prime}\right),
$$

where $R \equiv r / \Omega$ denotes (physical) areal radius.

An apparent horizon is given by the outermost radius $r$ at which $\theta_{+}=0$. A zero of this function is detected using a standard root finding algorithm (the Python routine scipy.optimize.brentq). Even before a zero forms, we can detect and track a minimum of $\theta_{+}$in order to have a better initial guess at where the zero is about to occur.

Once an apparent horizon has formed at a radius $r=r_{\mathrm{AH}}$ (with corresponding mass $M_{\mathrm{AH}}=\frac{1}{2} R_{\mathrm{AH}}$ ), we excise just inside it, at $r=0.9 r_{\mathrm{AH}} \equiv r_{\min }$. The numerical solution is interpolated (using cubic interpolation) to a new grid with inner boundary at $r=r_{\min }$, discarding the part of the solution at smaller values of $r$. The evolution is then continued on the new grid.

The optical scalars (101) are closely related to the Hawking mass

$$
M_{\mathrm{H}}=\frac{1}{2} R\left(1+R^{2} \theta_{+} \theta_{-}\right) .
$$

Its limit at $\mathscr{I}^{+}$is the Bondi mass $M_{\mathrm{B}}$, which will be a useful quantity to evaluate during our numerical evolutions. Although formally singular, the results of our regularity analysis (section 4.1.2) imply that the limit at $\mathscr{I}^{+}$of (102) is

$$
M_{\mathrm{B}}=\left.M_{\mathrm{H}}\right|_{\mathscr{I}+}=-\frac{3}{4} K^{-2}\left(K r^{5} \pi^{\prime \prime}+2 r^{3} \Omega^{(4)}\right) .
$$

Evaluating the fourth derivative of the conformal factor at $\mathscr{I}^{+}$is prone to numerical error; we have found it to be more accurate to extrapolate $M_{\mathrm{H}}$ from the interior.

\section{Numerical results}

\subsection{Initial data}

We start the evolution from initial data that are close to either Minkowski or Schwarzschild spacetime. The geometry variables $(\Omega, \pi, \tilde{N}$ and $X)$ are first set according to the respective vacuum solution, then initial data for the matter fields are specified, and finally the constraints and elliptic gauge conditions are re-solved for the geometry variables.

Schwarzschild spacetime in CMC coordinates is given by [4, 41]

$$
d s^{2}=-\left(1-\frac{2 M}{\bar{r}}\right) \mathrm{d} t^{2}+\frac{1}{f^{2}} \mathrm{~d} \bar{r}^{2}-\frac{2 a}{f} \mathrm{~d} t \mathrm{~d} \bar{r}+\bar{r}^{2}\left(\mathrm{~d} \theta^{2}+\sin ^{2} \theta \mathrm{d} \phi^{2}\right)
$$

where

$$
f(\bar{r})=\left(1-\frac{2 M}{\bar{r}}+a^{2}\right)^{1 / 2}, \quad a(\bar{r})=\frac{K \bar{r}}{3}-\frac{C}{\bar{r}^{2}},
$$

and $M$ (mass), $K$ (mean curvature) and $C$ are constants. The radial coordinate $\bar{r}$ needs to be transformed to a new radial coordinate $r$ such that the spatial metric is conformally flat in the new coordinates. This yields the ODE

$$
\frac{\mathrm{d} r}{\mathrm{~d} \bar{r}}=\frac{r}{\bar{r} f(\bar{r})} .
$$


Since the physical radial coordinate $\bar{r}$ has infinite range, it is more convenient to work with $s \equiv 1 / \bar{r}$ and set

$$
A(s) \equiv \frac{1}{3} K-C s^{3}, \quad F(s) \equiv s^{2}-2 M s^{3}+A(s)^{2} .
$$

Equation (106) now takes the form

$$
\frac{\mathrm{d} s}{\mathrm{~d} r}=\frac{-F(s)^{1 / 2}}{r} \text {. }
$$

First we determine the $r$-coordinate of the horizon by numerical integration,

$$
r_{\mathrm{H}}=\exp \left(-\int_{0}^{s_{H}} F(s)^{-1 / 2} \mathrm{~d} s\right)
$$

where $s_{H}=1 /(2 M)$. (Recall that we choose $\mathscr{I}^{+}$, i.e. $\bar{r}=\infty \Leftrightarrow s=0$, to correspond to $r=1$.) We place the excision boundary at $r_{\text {min }}=0.9 r_{\mathrm{H}}$. The ODE (108) is now solved numerically on the interval $r \in\left[r_{\min }, 1\right]$ with initial condition $s(1)=0$. (We use the Python routine scipy.integrate.odeint.)

In terms of the numerically determined function $s(r)$, the geometry variables are obtained as

$$
\Omega=r s, \quad \pi=2 C r^{-3} s^{2}, \quad \tilde{N}=r F(s)^{1 / 2}, \quad X=C s^{2}-\frac{1}{3} K .
$$

For $M=C=0$ this reduces to Minkowski spacetime (with $r \in[0,1]$ ),

$$
\Omega=\frac{1}{6} K\left(1-r^{2}\right), \quad \pi=0, \quad \tilde{N}=\frac{1}{6} K\left(1+r^{2}\right), \quad X=-\frac{1}{3} K .
$$

For the matter fields we consider three types of initial data,

(i) scalar field: specify initial data for $(\tilde{\phi}, \tilde{\psi})$, take the Yang-Mills field to vanish,

(ii) gravitational-sector Yang-Mills: specify initial data for $\left(F, D_{F}\right)$, all other fields vanish,

(iii) sphaleron-sector Yang-Mills: specify initial data for $\left(H, D_{H}\right)$, take $F=D_{F}=$ $\tilde{\phi}=\tilde{\psi}=0$, solve for $D_{L}$ and $G$.

Note that in case (iii), $F=D_{F}=0$ initially but not during the evolution. In each case, the initial data are chosen to be a Gaussian that is approximately ingoing initially, e.g. for the pair $(\tilde{\phi}, \tilde{\psi})$,

$$
\tilde{\phi}=A \exp \left(-\frac{\left(r-r_{0}\right)^{2}}{2 \sigma^{2}}\right), \quad \tilde{\psi}=\tilde{\phi}^{\prime}+r^{-1} \tilde{\phi} .
$$

For all the numerical evolutions shown here, we choose $r_{0}=0.5$ and $\sigma=0.05$.

If we add these initial data for the matter fields to the Minkowski background solution (re-solving the constraints), we obtain evolutions that either disperse or collapse to a black hole, depending on the amplitude $A$. If the background geometry is Schwarzschild, the matter will partly accrete onto the black hole.

\subsection{Scalar field evolutions}

We begin with a scalar field evolution that disperses to flat space (figure 10. The amplitude is chosen to be $A=0.6$, which corresponds to an initial Bondi mass $M_{\mathrm{B}}^{\mathrm{i}}=0.59$, already well in the non-linear regime. At late times, the field decays as a power law. At $\mathscr{I}^{+}, \tilde{\phi} \sim t^{-2}$, whereas at the origin and in fact at any finite radius, $\tilde{\phi} \sim t^{-3}$. These results are in agreement with [18. The fact that the scalar field decays more slowly at $\mathscr{I}^{+}$than away from $\mathscr{I}^{+}$leads to the solution becoming increasingly 


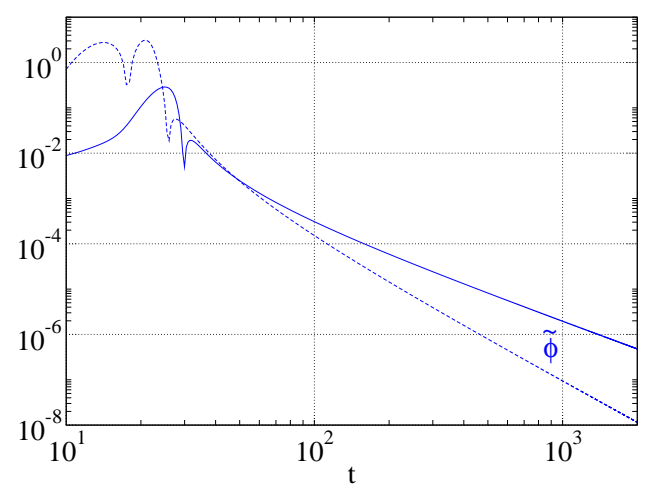

Figure 1. Scalar field dispersal. The initial Bondi mass is $M_{\mathrm{B}}^{\mathrm{i}}=0.59$. At $\mathscr{I}^{+}$(solid line) the field decays as $\tilde{\phi} \sim t^{-2}$ at late times. At the origin (dashed line), $\tilde{\phi} \sim t^{-3}$.

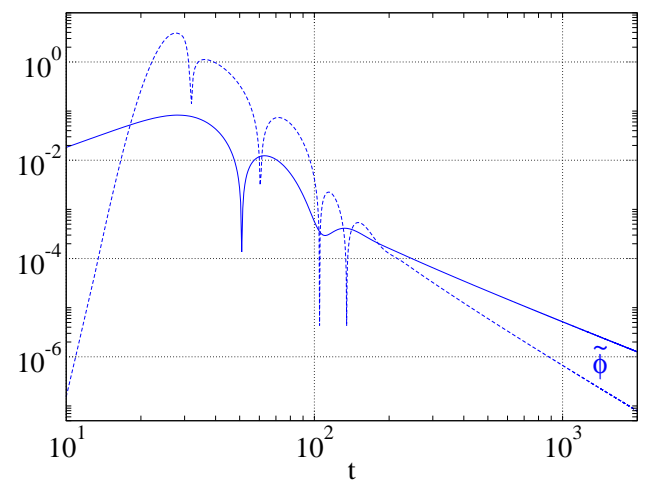

Figure 2. Scalar field accretion. The initial Bondi and apparent horizon masses are $M_{\mathrm{B}}^{\mathrm{i}}=1.45, M_{\mathrm{AH}}^{\mathrm{i}}=1$, and the final masses are $M_{\mathrm{B}}^{\mathrm{f}}=M_{\mathrm{AH}}^{\mathrm{f}}=1.44$. At $\mathscr{I}^{+}$(solid line) the field decays as $\tilde{\phi} \sim t^{-2}$ at late times. At the horizon (dashed line), $\tilde{\phi} \sim t^{-3}$.

peaked at $\mathscr{I}^{+}$, similar to the formation of a boundary layer. Eventually this feature cannot be resolved at a fixed numerical resolution. The simulations presented here were run at two different resolutions in order to make sure that the plots can be trusted during the times shown. For the simulations shown in the plots, $N=8000$ grid points were used.

Next we choose initial data containing a black hole with a scalar field perturbation (figure 21). Initially the Bondi mass is $M_{\mathrm{B}}^{\mathrm{i}}=1.45$ and the apparent horizon mass is $M_{\mathrm{AH}}^{\mathrm{i}}=1$. Almost all of the matter falls into the black hole - the final Bondi mass, which agrees with the final apparent horizon mass, is $M_{\mathrm{B}}^{\mathrm{f}}=M_{\mathrm{AH}}^{\mathrm{f}}=1.44$. At late times we observe a power-law decay of the scalar field with the same decay exponents as in the evolution that dispersed to flat space, where now instead of the origin, we evaluate the field at the horizon. These exponents agree with those found in the test field approximation [24.

Finally we return to the case of regular initial data as in the first simulation but increase the amplitude such that a black hole forms in the course of the evolution (figure 3). The initial Bondi mass is $M_{\mathrm{B}}^{\mathrm{i}}=2.74$, and the final Bondi and apparent 


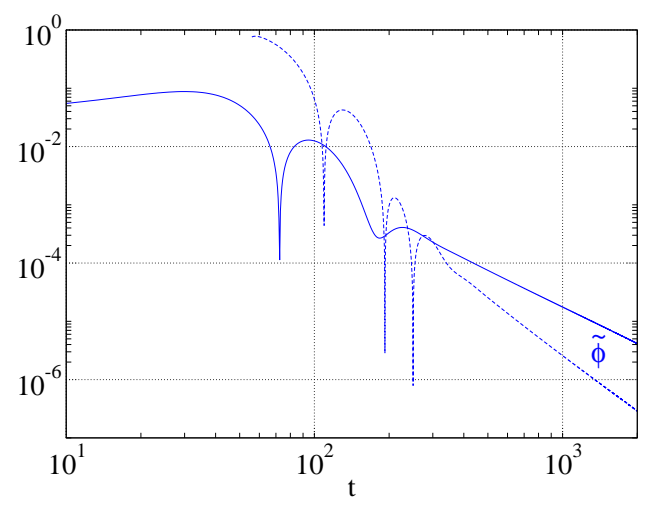

Figure 3. Scalar field collapse. The initial Bondi mass is $M_{\mathrm{B}}^{\mathrm{i}}=2.74$ and the final Bondi and apparent horizon masses are $M_{\mathrm{B}}^{\mathrm{f}}=M_{\mathrm{AH}}^{\mathrm{f}}=2.71$. At $\mathscr{I}^{+}$( solid line) the field decays as $\tilde{\phi} \sim t^{-2}$ at late times. At the horizon (after it forms, dashed line), $\tilde{\phi} \sim t^{-3}$.

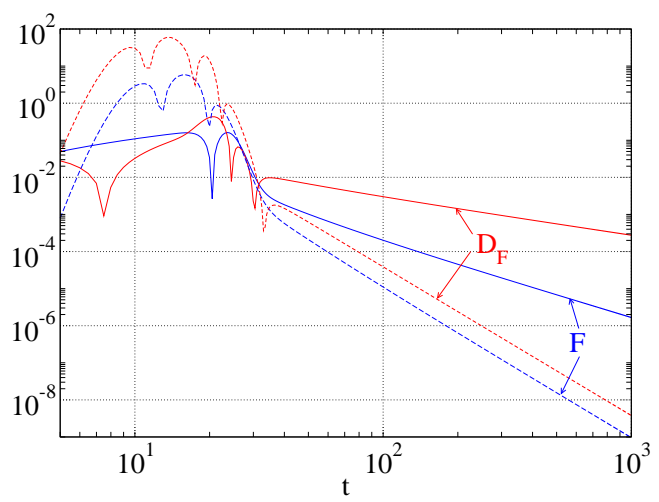

Figure 4. Gravitational-sector Yang-Mills dispersal. The initial Bondi mass is $M_{\mathrm{B}}^{\mathrm{i}}=0.63$. At $\mathscr{I}^{+}$(solid lines) the fields decay as $F \sim t^{-2}$ and $D_{F} \sim t^{-1}$ at late times. At the origin (dashed lines), $F \sim D_{F} \sim t^{-4}$.

horizon masses are $M_{\mathrm{B}}^{\mathrm{f}}=M_{\mathrm{AH}}^{\mathrm{f}}=2.71$. Again we find the same decay exponents as in the dispersing evolution.

\subsection{Gravitational-sector Yang-Mills evolutions}

Consider now the gravitational sector of Yang-Mills. As for the scalar field, we begin with an evolution that disperses to flat space (figure 44). We find that the potential $F$ decays as $F \sim t^{-2}$ at $\mathscr{I}^{+}$and $F \sim t^{-4}$ at the origin. These exponents agree with 23. Let us also evaluate the electric field $D_{F}$. Whereas this decays at the same rate as $F$ at finite radius, we observe a slower decay $D_{F} \sim t^{-1}$ at $\mathscr{I}^{+}$. This may seem surprising at first but can be explained as follows. Consider the evolution equation (75) for $F$,

$$
\dot{F}=r X F^{\prime}+2 X F-\tilde{N} D_{F} .
$$

Notice the radial derivative $F^{\prime}$ on the right-hand side. Suppose $F^{\prime}$ decayed at the same rate as $F$ at $\mathscr{I}^{+}$. Then, by continuity, $F$ would decay at the same rate in a 


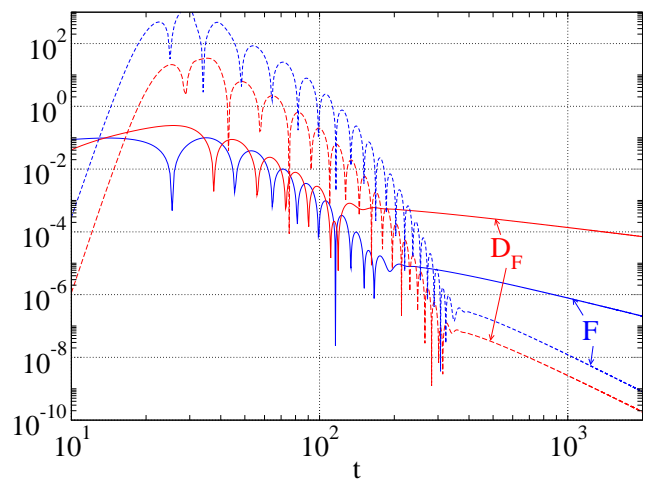

Figure 5. Gravitational-sector Yang-Mills accretion. The initial Bondi and apparent horizon masses are $M_{\mathrm{B}}^{\mathrm{i}}=1.49$ and $M_{\mathrm{AH}}^{\mathrm{i}}=1$, and the final masses are $M_{\mathrm{B}}^{\mathrm{f}}=M_{\mathrm{AH}}^{\mathrm{f}}=1.45$. At $\mathscr{I}^{+}$(solid lines) the fields decay as $F \sim t^{-2}$ and $D_{F} \sim t^{-1}$ at late times. At the horizon (dashed lines), $F \sim D_{F} \sim t^{-4}$.

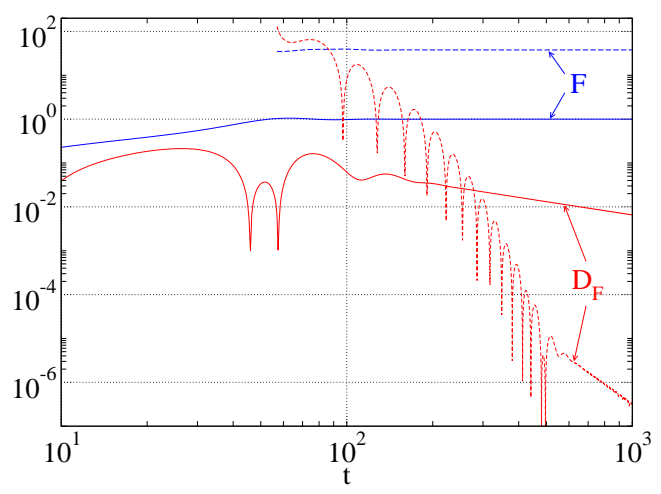

Figure 6. Gravitational-sector Yang-Mills collapse. The initial Bondi mass is $M_{\mathrm{B}}^{\mathrm{i}}=3.04$ and the final Bondi and apparent horizon masses are $M_{\mathrm{B}}^{\mathrm{f}}=M_{\mathrm{AH}}^{\mathrm{f}}=$ 2.49. The final coordinate location of the apparent horizon is $r_{\mathrm{AH}}=0.163$. At $\mathscr{I}^{+}$(solid lines) $F \rightarrow-1$ and $D_{F} \sim t^{-1}$ at late times. At the horizon (after it forms, dashed lines), $F \rightarrow-37.9 \approx-r_{\mathrm{AH}}^{-2}$ and $D_{F} \sim t^{-4}$.

neighbourhood of $\mathscr{I}^{+}$. But we know from previous work 24, 23] - and our numerical evolutions suggest this too- that $F$ decays faster at any point away from $\mathscr{I}^{+}$than at $\mathscr{I}^{+}$itself. Hence by contradiction $F^{\prime}$ must decay at a slower rate at $\mathscr{I}^{+}$than $F$. From (113) we deduce that $D_{F}$ must also decay at that slower rate. (This argument cannot determine the precise decay rate of $D_{F}$ at $\mathscr{I}^{+}$, it merely shows that it must decay more slowly than $F$.)

Next we consider accretion onto a black hole (figure 5). The decay exponents found in this case are the same as in the dispersing evolution and agree with the test field approximation 24 .

Finally we turn to collapse (figure 60). While the electric field decays at the same rates as above, the behaviour of the potential $F$ is different. At late times this approaches the static solution $F=2 /\left(g r^{2}\right)$ (recall we set $g=-2$ ). We shall see in section 6.5 that this is another vacuum solution of the Yang-Mills equations (in addition to $F=0)$. 

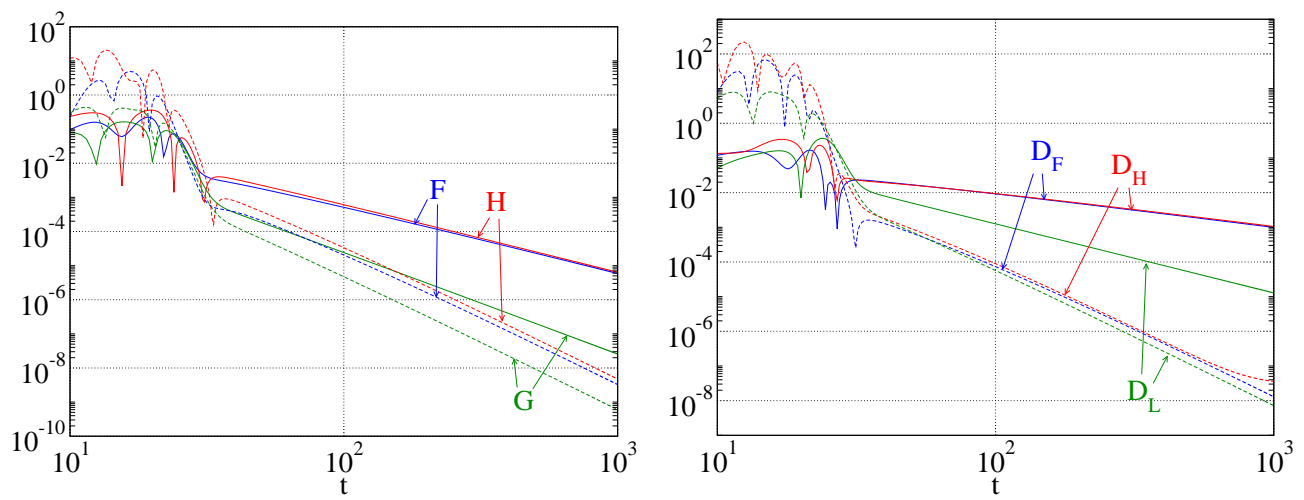

Figure 7. Sphaleron-sector Yang-Mills dispersal. The initial Bondi mass is $M_{\mathrm{B}}^{\mathrm{i}}=0.75$. At $\mathscr{I}+$ (solid lines) the fields decay as $F \sim H \sim t^{-2}, G \sim t^{-3}$, $D_{F} \sim D_{H} \sim t^{-1}$ and $D_{L} \sim t^{-2}$. At the origin (dashed lines), $F \sim H \sim G \sim$ $D_{F} \sim D_{H} \sim D_{L} \sim t^{-4}$.
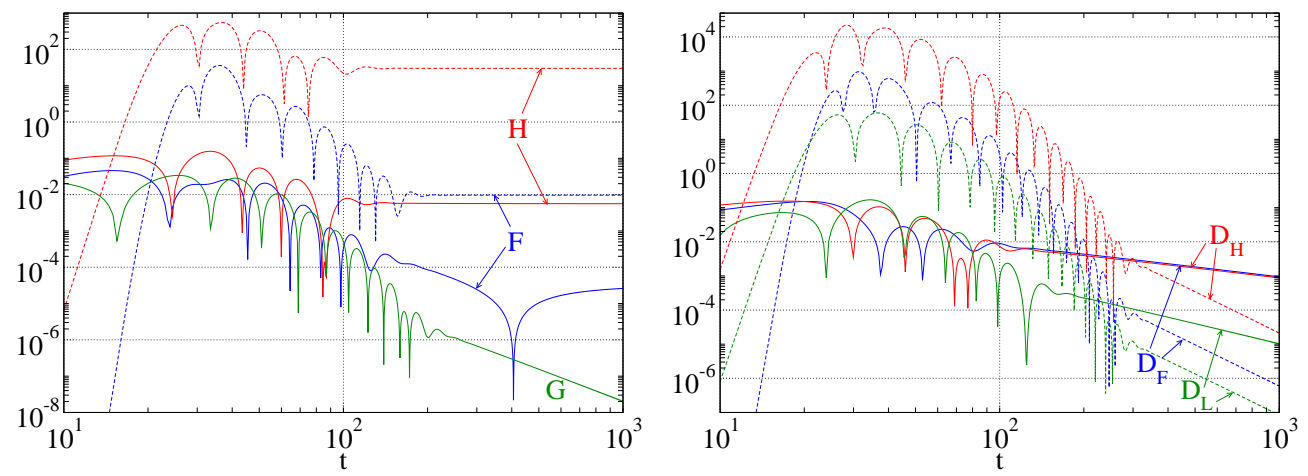

Figure 8. Sphaleron-sector Yang-Mills accretion. The initial Bondi and apparent horizon masses are $M_{\mathrm{B}}^{\mathrm{i}}=1.61$ and $M_{\mathrm{AH}}^{\mathrm{i}}=1$, and the final masses are $M_{\mathrm{B}}^{\mathrm{f}}=M_{\mathrm{AH}}^{\mathrm{f}}=2.49$. At $\mathscr{I}+$ (solid lines), $F$ and $H$ approach constants, $G \sim t^{-3}$, $D_{F} \sim D_{H} \sim t^{-1}$ and $D_{L} \sim t^{-2}$. At the horizon (dashed lines), $F$ and $H$ approach constants, $G$ is frozen to its initial value $G=0$, and $D_{F} \sim D_{H} \sim D_{L} \sim t^{-4}$.

\subsection{Sphaleron-sector Yang-Mills evolutions}

In the last set of evolutions we consider initial data of type (iii) (section 6.1) that include the sphaleron sector of Yang-Mills. Figure 7 shows a dispersing evolution. All fields decay. The potentials $F$ and $H$ decay at the same rate as in the gravitational sector, i.e., $F \sim H \sim t^{-2}$ at $\mathscr{I}^{+}$and $F \sim H \sim t^{-4}$ at the origin. The "gauge potential" $G$ decays as $G \sim t^{-3}$ at $\mathscr{I}^{+}$and $G \sim t^{-4}$ at the origin. The electric field components $D_{F}$ and $D_{H}$ also decay at the same rates as in the gravitational sector, i.e., $D_{F} \sim D_{H} \sim t^{-1}$ at $\mathscr{I}^{+}$and $D_{F} \sim D_{H} \sim t^{-4}$ at the origin. The component $D_{L}$ has a different decay at $\mathscr{I}^{+}, D_{L} \sim t^{-2}$, while $D_{L} \sim t^{-4}$ at the origin as for the other components.

Already in the accretion evolution (figure 8), a different behaviour is seen for the connection components. Now $F$ and $H$ approach a nonzero static solution at late times. The gauge potential $G$ is frozen to its initial value at the excision boundary, which for the initial data we choose is $G=0$ to within numerical roundoff. At $\mathscr{I}^{+}$, 

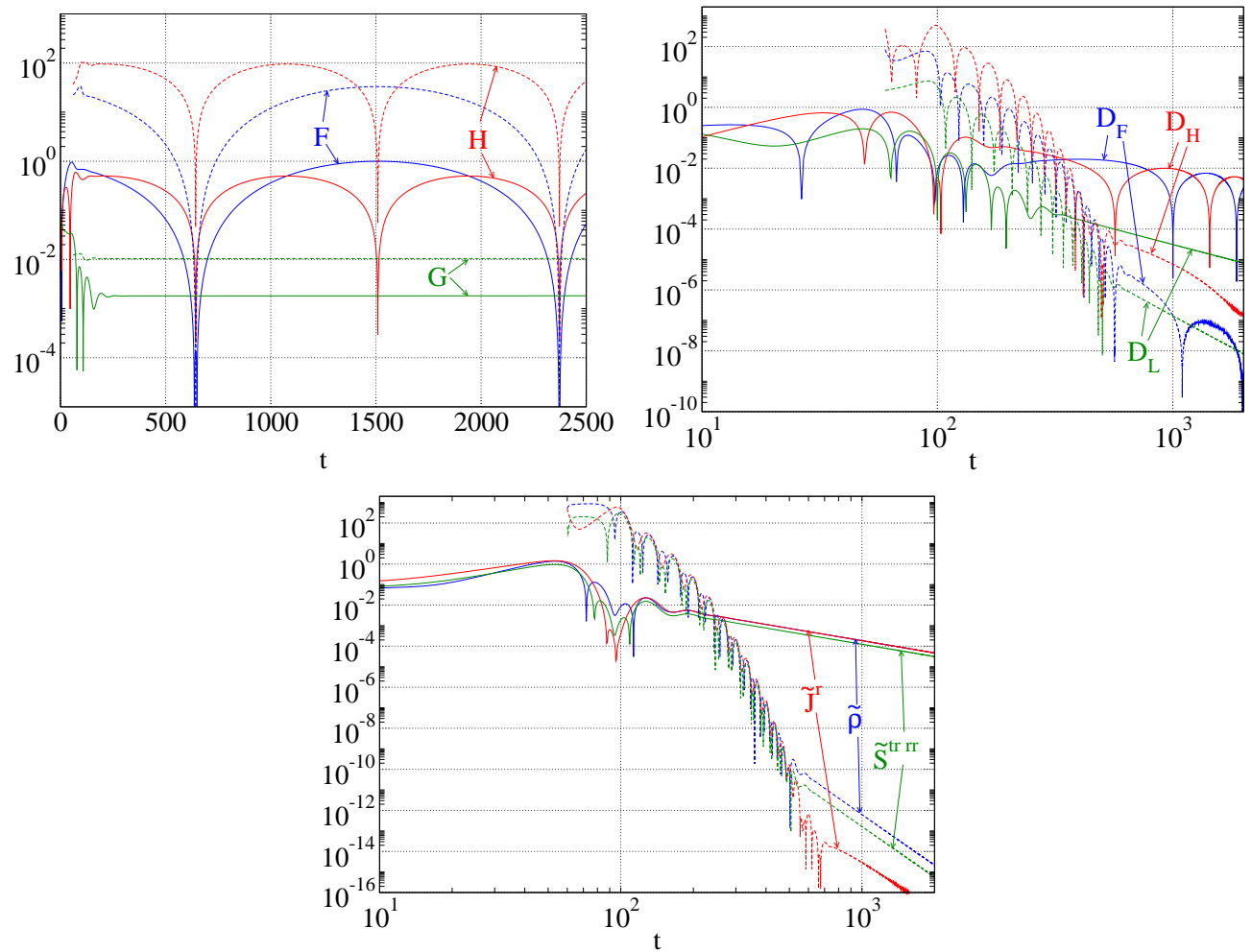

Figure 9. Sphaleron-sector Yang-Mills collapse. The initial Bondi mass is $M_{\mathrm{B}}^{\mathrm{i}}=$ 3.51 and the final Bondi and apparent horizon masses are $M_{\mathrm{B}}^{\mathrm{f}}=M_{\mathrm{AH}}^{\mathrm{f}}=2.67$. As before, solid lines refer to quantities observed at $\mathscr{I}^{+}$and dashed lines to quantities observed at the horizon after it forms. The top left panel shows that $G$ becomes asymptotically time independent, $G \rightarrow G_{0}=0.00183$ at $\mathscr{I}^{+}$, and $F$ and $H$ perform harmonic oscillations with period $T=1717 \approx 2 \pi /\left|g G_{0}\right|$. In the top right panel, $D_{F}$ and $D_{H}$ decay in damped harmonic oscillations, $D_{L} \sim t^{-2}$ at $\mathscr{I}^{+}$and $D_{L} \sim t^{-4}$ at the horizon. The bottom panel demonstrates that all independent components $\tilde{J}^{r}, \tilde{\rho}$ and $\tilde{S}^{\operatorname{tr} r r}$ of the energy-momentum tensor decay as $t^{-2}$ at $\mathscr{I}^{+}$and $t^{-8}$ at the horizon.

$G \sim t^{-3}$ as previously in the dispersing evolution. The decay of the eletric field is also the same as in the dispersing evolution.

Finally we study sphaleron-sector collapse (figure 9). Recall that we freeze the value of $G$ at the excision boundary once the apparent horizon forms. This results in the entire function $G$ becoming time independent (but nonzero). Surprisingly however, the potentials $F$ and $H$ do not become time independent but show a sinusoidal time dependence with a period close to $T=2 \pi /\left|g G_{0}\right|$, where $G_{0}$ is the asymptotic value of $G$ at $\mathscr{I}^{+}$. This peculiar behaviour will be explained in section 6.5 A first hint at what is happening can be found by evaluating the components of the energy-momentum tensor. Figure 9 demonstrates that all of these decay at the rate $t^{-2}$ at $\mathscr{I}^{+}$and $t^{-8}$ at the horizon, in accordance with the electric field decaying as $t^{-1}$ at $\mathscr{I}^{+}$and $t^{-4}$ at the horizon (recall that the energy-momentum tensor is quadratic in the field strength tensor). Hence a vacuum solution is approached. 


\subsection{The Yang-Mills vacuum}

The findings of the previous subsection suggest that we need to obtain a better understanding of the vacuum solutions to the Yang-Mills equations. Let us therefore take the field strength tensor to vanish, i.e., $D_{F}=D_{H}=D_{L}=B_{F}=B_{H}=B_{L}=0$. Equation (83) for the magnetic field implies

$$
r H^{\prime}+3 H=0 \Rightarrow H=H_{0}(t) r^{-3} .
$$

The linear combination $r^{2} \times 84$-850 yields

$$
r F^{\prime}+2 F=0 \Rightarrow F=F_{0}(t) r^{-2} .
$$

The radial gauge condition (89) implies

$$
r G^{\prime}+G=0 \Rightarrow G=G_{0}(t) r^{-1} .
$$

Since we freeze $G$ at the inner boundary after excision, we are only interested in the case where $G_{0}$ is a constant. Substituting (114)-(116) into the evolution equations (75) and (76) for $F$ and $H$, we obtain

$$
\dot{F}_{0}=-g G_{0} H_{0}, \quad \dot{H}_{0}=-G_{0}+g G_{0} F_{0} .
$$

The general solution to this pair of ODEs is

$$
F_{0}=g^{-1}+\alpha \sin \left(g G_{0} t+\phi\right), \quad H_{0}=-\alpha \cos \left(g G_{0} t+\phi\right) .
$$

The constant $\alpha$ is not arbitrary, however. In deriving (117) we used only one particular linear combination of (84) and (85). Substituting the solution (118) in either (84) or (85) fixes $\alpha=g^{-1}$. The form of the solution (118) agrees well with the numerical results in figure 9. We see that it is $G_{0}$ (the value of $G$ at $\mathscr{I}^{+}$) that determines the period of the oscillations. If $G$ is zero as in the final state of the accretion evolution, (118) implies that $F_{0}$ and $H_{0}$ are constants related by

$$
\left(1-g F_{0}\right)^{2}+\left(g H_{0}\right)^{2}=1 .
$$

If in addition $H_{0}=0$ as in the gravitational-sector evolutions, this implies $F_{0}=0$ or $F_{0}=2 / g$, i.e., by (115),

$$
F=0 \text { or } F=\frac{2}{g r^{2}} \text {. }
$$

In the Abelian-gauge version (90) of the gravitational-sector Yang-Mills ansatz, these two copies of the Yang-Mills vacuum correspond to $w= \pm 1$.

\section{Conclusions}

The purpose of this paper was to show how matter can be included in a hyperboloidal evolution scheme for the Einstein equations, and to implement such a scheme numerically in order to study power-law tails of matter fields up to future null infinity.

We assumed that the energy-momentum tensor is tracefree, for then the energymomentum conservation equations are conformally invariant and thus regular at null infinity. A large class of radiative matter models are included under this assumption. How to deal with non-tracefree matter in the conformal setting remains an open question. Of course, if the matter is such that it remains bounded away from $\mathscr{I}^{+}$during the evolution then one can live with matter field equations that are singular at $\mathscr{I}^{+}$as they never need to be evaluated there. 
We worked with a constrained ADM formulation of the Einstein equations on CMC slices 3. In that paper we showed (in the vacuum case) how the formally singular terms in the evolution equations at $\mathscr{I}^{+}$can in fact be evaluated in a regular way. In the present paper we showed that our analysis is unaffected by the addition of matter (section 2.3).

Two matter models were studied in detail, namely a conformally coupled scalar field and a Yang-Mills field. In both cases the energy-momentum tensor is tracefree and the matter equations of motion are conformally invariant. The use of conformal coupling instead of minimal coupling for the scalar field is essential here. We first derived the matter evolution equations and the source terms appearing in the Einstein equations without any symmetry assumptions. Subsequently we reduced the Einsteinmatter systems to spherical symmetry in isotropic coordinates. We worked with the most general ansatz (73) for the spherically symmetric Yang-Mills connection [33. 34, 35]; this is more general than the purely magnetic or gravitational-sector ansatz that is often used.

Our motivation to study spherically symmetric Einstein-matter evolutions on hyperboloidal slices arose partially from an earlier numerical study by one of the authors [5] that considered vacuum axisymmetric spacetimes. Whereas long-term stable evolutions of perturbed black holes could be achieved and the quasi-normal mode radiation correctly reproduced, we were unable to resolve the power-law tail of the gravitational field expected at late times. In the current spherically symmetric study a much higher numerical resolution could be used so that the tails could indeed be resolved. Otherwise the numerical method based on fourth-order finite differences is very similar to the one used in $\mathbf{5}$. One difference is that we are now able to handle a regular centre as well so that gravitational collapse can be studied; in [5] we only considered black hole evolutions with excised interior.

A general feature of tails in hyperboloidal evolutions is that the fields decay at a slower rate at $\mathscr{I}^{+}$than at finite radius. This means that the solution becomes increasingly peaked at $\mathscr{I}^{+}$, akin to the formation of a boundary layer. This is very challenging numerically - at a fixed numerical resolution the code is ultimately unable to resolve the solution. A possible direction for future development would be to introduce a time-dependent radial mapping so that an increasingly higher resolution can be provided near $\mathscr{I}^{+}$as the evolution proceeds. A similar adaptivity will be needed in order to study the formation of very small black holes in critical collapse. The current code already contains an (albeit time-independent) radial map in order to better resolve the steep gradients near the horizon of a black hole.

For scalar field matter our results are consistent with evolutions of the Einsteinscalar field system in Bondi coordinates [18. Our study goes further though because unlike Bondi coordinates, the coordinates we use can penetrate black hole horizons. Both for an evolution that starts out with a perturbed black hole and for an evolution that forms a black hole from regular initial data, we find the same decay exponents at $\mathscr{I}^{+}$and at finite radius as in the dispersing case, which agree with results obtained in the test field approximation 24.

For evolutions of the gravitational sector of Yang-Mills, the decay rates we found for the Yang-Mills connection in an evolution that disperses to flat space agree with evolutions in Bondi coordinates [23. For a Schwarzschild black hole with a YangMills perturbation we obtain the same decay rates, which in turn agree with the test field approximation 24. Somewhat different behaviour is seen in an evolution that collapses to a black hole from regular initial data. Here the Yang-Mills connection 
approaches a static solution that corresponds to another copy of the Yang-Mills vacuum.

A fact that does not seem to have been noted before is that in all three cases (dispersal, accretion and collapse), the electric field has a slower decay at $\mathscr{I}^{+}$than the connection, $D_{F} \sim t^{-1}$ as opposed to $F \sim t^{-2}$. At finite radius the decay rates agree, $F \sim D_{F} \sim t^{-4}$. Since the electric field is the physically measurable quantity, it seems more natural to consider its decay rather than that of the connection.

As far as we know we presented the first dynamical numerical evolutions that include the sphaleron sector of Yang-Mills, i.e., the fully general ansatz (73) for the spherically symmetric Yang-Mills connection. In an evolution that disperses to Minkowski space, both potentials $F$ and $H$ appearing in the ansatz for the connection decay at the same rates as for the gravitational-sector evolutions. For an accretion evolution, however, both potentials approach nonzero static solutions, and in a collapse evolution they become sinusoidal functions of time. Nevertheless, in all cases a vacuum solution is approached. We were able to explain this behaviour by deriving the general vacuum solution to the Yang-Mills equations in our setting.

The nontrivial structure of the Yang-Mills vacuum gives rise to interesting threshold behaviour [42. The Einstein-Yang-Mills system exhibits remarkably rich dynamics [37, 36] due to the existence of nontrivial asymptotically flat static solutions 43. 44. We hope to investigate some of these phenomena further using our hyperboloidal evolution code.

\section{Acknowledgments}

The authors would like to thank Lars Andersson, Piotr Bizoń, Michael Pürrer, István Rácz, Olivier Sarbach, Christian Schell and Anıl Zenginoğlu for helpful discussions. O.R. gratefully acknowledges support from the German Research Foundation (DFG) through a Heisenberg Fellowship and research grant RI 2246/2. V.M. was supported by NSF grant PHY-0963869 to Yale University. V.M. is grateful to the Albert Einstein Institute (Potsdam), the Erwin Schrödinger Institute and the University of Vienna for hospitality and support during the course of some of this work.

\section{Appendix A. Conformal transformations and $3+1$ decompositions}

In this appendix we collect a few useful identities used throughout the paper.

Appendix A.1. Conformal transformation of the connection and curvature

Consider a conformal transformation of the metric,

$$
\gamma_{a b}=\Omega^{2} g_{a b}
$$

The following applies to any dimension $n$ and any signature (e.g. the spacetime metric or the induced spatial metric on the $t=$ const slices), and hence we use general indices $a, b, \ldots$ to indicate this.

Let $\Gamma_{b c}^{a}$ denote the Christoffel symbols of the metric connection $\nabla$ of $g$ and $\tilde{\Gamma}^{a} b c$ the Christoffel symbols of the metric connection $\tilde{\nabla}$ of $\gamma$. They are related by

$$
\Gamma_{b c}^{a}=\tilde{\Gamma}_{b c}^{a}-\Omega^{-1}\left(\delta_{b}^{a} \Omega_{, c}+\delta_{c}^{a} \Omega_{, b}-\gamma_{b c} \gamma^{a d} \tilde{\nabla}_{d} \Omega\right) .
$$


Hyperboloidal Einstein-matter evolution and tails for scalar and Yang-Mills fields 26

The Ricci tensor transforms as

$$
\begin{aligned}
R_{a b}= & \tilde{R}_{a b}+(n-2) \Omega^{-1} \tilde{\nabla}_{a} \tilde{\nabla}_{b} \Omega \\
& +\gamma_{a b} \gamma^{c d}\left[\Omega^{-1} \tilde{\nabla}_{c} \tilde{\nabla}_{d} \Omega-(n-1) \Omega^{-2} \Omega_{, c} \Omega, d\right]
\end{aligned}
$$

and the Ricci scalar as

$$
R=\Omega^{2} \tilde{R}+2(n-1) \Omega \gamma^{a b} \tilde{\nabla}_{a} \tilde{\nabla}_{b} \Omega-n(n-1) \gamma^{a b} \Omega_{, a} \Omega_{, b} .
$$

Appendix A.2. Physical and conformal extrinsic curvature

The physical extrinsic curvature $K_{i j}$ and the unphysical extrinsic curvature $C_{i j}$ are defined as

$$
K_{i j}=-\frac{1}{2} \mathcal{L}_{n} g_{i j}, \quad C_{i j}=-\frac{1}{2} \mathcal{L}_{\tilde{n}} \gamma_{i j} .
$$

By translating the right-hand side of the first equation into conformal language and comparing with the second, we find for the traceless part

$$
C_{i j}^{\mathrm{tr}}=\Omega K_{i j}^{\mathrm{tr}}=-\mu_{\gamma}^{-1} \gamma_{i k} \gamma_{j l} \pi^{\mathrm{tr} k l} .
$$

Taking instead the trace, we recover equation (16),

$$
\mathcal{L}_{\tilde{n}} \Omega=-\frac{1}{3}(K+\Omega C),
$$

where $C \equiv \gamma^{i j} C_{i j}$ and we recall our convention $K \equiv-g^{i j} K_{i j}=$ const $>0$.

\section{Appendix A.3. $3+1$ decomposition of the scalar Hessian}

The projections of the covariant Hessian ${ }^{(4)} \tilde{\nabla}_{\mu}{ }^{(4)} \tilde{\nabla}_{\nu} \tilde{\phi}$ of a conformal scalar field $\tilde{\phi}$ can be written in $3+1$ form as

$$
\begin{aligned}
n^{\mu} n^{\nu}{ }^{(4)} \tilde{\nabla}_{\mu}{ }^{(4)} \tilde{\nabla}_{\nu} \tilde{\phi} & =\mathcal{L}_{\tilde{n}}^{2} \tilde{\phi}-\tilde{N}^{-1} \gamma^{i j} \tilde{N}_{, i} \tilde{\phi}_{, j} \\
\gamma^{i \mu} n^{\nu}{ }^{(4)} \tilde{\nabla}_{\mu}{ }^{(4)} \tilde{\nabla}_{\nu} \tilde{\phi} & =\partial^{i} \mathcal{L}_{\tilde{n}} \tilde{\phi}+C^{i j} \tilde{\phi}_{, j} \\
\gamma_{i}{ }^{\mu} \gamma_{j}{ }^{\nu}{ }^{(4)} \tilde{\nabla}_{\mu}{ }^{(4)} \tilde{\nabla}_{\nu} \tilde{\phi} & =\tilde{\nabla}_{i} \tilde{\nabla}_{j} \tilde{\phi}+C_{i j} \mathcal{L}_{\tilde{n}} \tilde{\phi}
\end{aligned}
$$

Appendix A.4. $3+1$ decomposition of the conformal spacetime Ricci tensor

One of the equations of a $3+1$ decomposition in the conformal spacetime is

$\gamma_{i}{ }^{\mu} \gamma_{j}{ }^{\nu}{ }^{(4)} \tilde{R}_{\mu \nu}=-\mathcal{L}_{\tilde{n}} C_{i j}-2 \gamma^{k l} C_{i k} C_{j l}+C_{i j} C-\tilde{N}^{-1} \tilde{\nabla}_{i} \tilde{\nabla}_{j} \tilde{N}+\tilde{R}_{i j}$.

Separating the trace and traceless part of $C_{i j}$ and using (A.6), we can write the traceless part of (A.11) as

$$
\begin{aligned}
{\left[\gamma^{i \mu} \gamma^{j \nu(4)} \tilde{R}_{\mu \nu}\right]^{\operatorname{tr}} } & =\mu_{\gamma}^{-1} \mathcal{L}_{\tilde{n}} \pi^{\operatorname{tr} i j}+2 \mu_{\gamma}^{-2} \gamma_{k l} \pi^{\operatorname{tr} i k} \pi^{\operatorname{tr} j l}-\frac{2}{3} C \mu_{\gamma}^{-1} \pi^{\operatorname{tr} i j} \\
& -\tilde{N}^{-1}\left[\tilde{\nabla}^{i} \tilde{\nabla}^{j} \tilde{N}\right]^{\operatorname{tr}}+\tilde{R}^{\operatorname{tr} i j} .
\end{aligned}
$$

Note the similarity of (A.12) with (18); formally it can be obtained from that equation by setting $\Omega \equiv 1$, replacing $K$ with $-C$ and noting that the source term now refers to the conformal geometry. Taking instead the trace of (A.11) we find

$$
\gamma^{\mu \nu(4)} \tilde{R}_{\mu \nu}=-\tilde{N}^{-1} \tilde{\nabla}^{i} \tilde{\nabla}_{i} \tilde{N}+\tilde{R}+C^{2}-\mathcal{L}_{\tilde{n}} C .
$$

We also have the Gauss-Codazzi equation analogous to the Hamiltonian constraint (20),

$$
\begin{aligned}
\tilde{n}^{\mu} \tilde{n}^{\nu(4)} \tilde{R}_{\mu \nu}+\gamma^{\mu \nu(4)} \tilde{R}_{\mu \nu}=2 \tilde{n}^{\mu} \tilde{n}^{\nu(4)} \tilde{G}_{\mu \nu} \\
=\tilde{R}+C^{2}-\gamma^{i k} \gamma^{j l} C_{i j} C_{k l}=\tilde{R}+\frac{2}{3} C^{2}-\mu_{\gamma}^{-2} \gamma_{i k} \gamma_{j l} \pi^{\operatorname{tr} i j} \pi^{\operatorname{tr} k l} .
\end{aligned}
$$


Taking linear combinations of A.13 and A.14 we obtain

$\tilde{n}^{\mu} \tilde{n}^{\nu(4)} \tilde{R}_{\mu \nu}=\tilde{N}^{-1} \tilde{\nabla}^{i} \tilde{\nabla}_{i} \tilde{N}-\frac{1}{3} C^{2}-\mu_{\gamma}^{-2} \gamma_{i k} \gamma_{j l} \pi^{\operatorname{tr} i j} \pi^{\operatorname{tr} k l}+\mathcal{L}_{\tilde{n}} C$,

(4) $\tilde{R}=\gamma^{\mu \nu(4)} \tilde{R}_{\mu \nu}-\tilde{n}^{\mu} \tilde{n}^{\nu(4)} \tilde{R}_{\mu \nu}$

$$
=-2 \tilde{N}^{-1} \tilde{\nabla}^{i} \tilde{\nabla}_{i} \tilde{N}+\tilde{R}+\frac{4}{3} C^{2}+\mu_{\gamma}^{-2} \gamma_{i k} \gamma_{j l} \pi^{\operatorname{tr} i j} \pi^{\operatorname{tr} k l}-2 \mathcal{L}_{\tilde{n}} C .
$$

Finally, the Gauss-Codazzi equation analogous to the momentum constraint (21) is

$$
\gamma^{i \mu} \tilde{n}^{\nu(4)} \tilde{R}_{\mu \nu}=\mu_{\gamma}^{-1} \tilde{\nabla}_{j} \pi^{\operatorname{tr} i j}+\frac{2}{3} \gamma^{i j} C_{, j}
$$

\section{References}

[1] Sarbach O and Tiglio M 2012 Continuum and discrete initial-boundary value problems and Einstein's field equations Living Rev. Relativity 15(9)

[2] Penrose R 1965 Zero rest-mass fields including gravitation: Asymptotic behaviour Proc. Royal Soc. London A 284 159-203

[3] Moncrief V and Rinne O 2009 Regularity of the Einstein equations at future null infinity Class. Quantum Grav. 26125010

[4] Arnowitt R, Deser S and Misner C W 1962 The dynamics of general relativity Gravitation: an introduction to current research ed Witten L (New York: Wiley) chap 7

[5] Rinne O 2010 An axisymmetric evolution code for the Einstein equations on hyperboloidal slices Class. Quantum Grav. 27035014

[6] Friedrich H 1983 Cauchy problems for the conformal vacuum field equations in general relativity Commun. Math. Phys. 91 445-472

[7] Frauendiener J 2004 Conformal infinity Living Rev. Relativity 7(1)

[8] Husa S 2002 Problems and successes in the numerical approach to the conformal field equations Lect. Notes Phys. 604 239-260

[9] Husa S 2003 Numerical relativity with the conformal field equations Lect. Notes Phys. 617 159-192

[10] Hübner P 1995 General relativistic scalar-field models and asymptotic flatness Class. Quantum Grav. 12 791-808

[11] Hübner P 1996 A method for calculating the structure of (singular) spacetimes in the large Phys. Rev. D 53 701-721

[12] Zenginoğlu A 2008 Hyperbolodial evolution with the Einstein equations Class. Quantum Grav. 25195025

[13] Bardeen J M, Sarbach O and Buchman L T 2011 Tetrad formalism for numerical relativity on conformally compactified constant mean curvature hypersurfaces Phys. Rev. D $\mathbf{8 3} 104045$

[14] Buchman L T, Pfeiffer H P and Bardeen J M 2009 Black hole initial data on hyperboloidal slices Phys. Rev. D $\mathbf{8 0} 084024$

[15] Bardeen J M and Buchman L T 2012 Bondi-Sachs energy-momentum for the constant mean extrinsic curvature initial value problem Phys. Rev. D 85064035

[16] Price R H 1972 Nonspherical perturbations of relativistic gravitational collapse. I. scalar and gravitational perturbations Phys. Rev. D 5 2419-2439

[17] Dafermos M and Rodnianski I 2005 A proof of Price's law for the collapse of a self-gravitating scalar field Inventiones Mathematicae 162 381-457

[18] Pürrer M, Husa S and Aichelburg P C 2005 News from critical collapse: Bondi mass, tails, and quasinormal modes Phys. Rev. D 71104005

[19] Leaver E W 1986 Spectral decomposition of the perturbation response of the Schwarzschild geometry Phys. Rev. D 34 384-408

[20] Gundlach C, Price R H and Pullin J 1994 Late-time behavior of stellar collapse and explosions. I. linearized perturbations Phys. Rev. D 49 883-889

[21] Gundlach C, Price R H and Pullin J 1994 Late-time behavior of stellar collapse and explosions. II. nonlinear evolution Phys. Rev. D 49 890-899

[22] Bizoń P, Chmaj T and Rostworowski A 2007 Late-time tails of a Yang-Mills field on Minkowski and Schwarzschild backgrounds Class. Quantum Grav. 24 F55-F63

[23] Pürrer M and Aichelburg P C 2009 Tails for the Einstein-Yang-Mills system Class. Quantum Grav. 26035004

[24] Zenginoğlu A 2008 A hyperboloidal study of tail decay rates for scalar and Yang-Mills fields Class. Quantum Grav. 25175013 
Hyperboloidal Einstein-matter evolution and tails for scalar and Yang-Mills fields 28

[25] Friedrich H 1991 On the global existence and the asymptotic behavior of solutions to the EinsteinMaxwell-Yang-Mills equations J. Diff. Geom. 34 275-345

[26] Lübbe C and Valiente Kroon J A 2011 A conformal approach for the analysis of the non-linear stability of pure radiation cosmologies Preprint http://www.arxiv.org/abs/1111.4691

[27] Winicour J 1988 Massive fields at null infinity J. Math. Phys. 29 2117-2121

[28] York Jr J W 1979 Kinematics and dynamics of general relativity Sources of gravitational radiation ed Smarr L L (Cambridge University Press) pp 83-126

[29] Andersson L, Chruściel P T and Friedrich H 1992 On the regularity of solutions to the Yamabe equation and the existence of smooth hyperboloidal initial data for Einstein's field equations Commun. Math. Phys. 149 587-612

[30] Bekenstein J D 1974 Exact solutions of Einstein-conformal scalar equations Ann. Phys. 82 $535-547$

[31] Rinne O and Stewart J M 2005 A strongly hyperbolic and regular reduction of Einstein's equations for axisymmetric spacetimes Class. Quantum Grav. 22 1143-1166

[32] Ruiz M, Alcubierre M and Núñez D 2007 Regularization of spherical and axisymmetric evolution codes in numerical relativity Gen. Relativ. Gravit. 40 159-182

[33] Witten E 1977 Some exact multipseudoparticle solutions of classical Yang-Mills theory Phys. Rev. Lett. 38 121-124

[34] Gu C and Hu H 1981 On the spherically symmetric gauge fields Commun. Math. Phys. 79 75-90

[35] Sarbach O 2000 On the generalization of the Regge-Wheeler equation for self-gravitating matter fields $\mathrm{PhD}$ thesis University of Zurich

[36] Choptuik M W, Hirschmann E W and Marsa R L 1999 New critical behavior in Einstein-YangMills collapse Phys. Rev. D 60124011

[37] Choptuik M W, Chmaj T and Bizoń P 1996 Critical behavior in gravitational collapse of a Yang-Mills field Phys. Rev. Lett. 77 424-427

[38] Fodor G and Rácz I 2008 Numerical investigation of highly excited magnetic monopoles in SU(2) Yang-Mills-Higgs theory Phys. Rev. D 77025019

[39] Kreiss H O and Oliger J 1973 Methods for the approximate solution of time dependent problems Global Atmospheric Research Programme (Publication Series No. 10)

[40] Brill D R, Cavallo J M and Isenberg J A 1980 K-surfaces in the Schwarzschild space-time and the construction of lattice cosmologies J. Math. Phys. 21 2789-2796

[41] Malec E and Ó Murchadha N 2003 Constant mean curvature slices in the extended Schwarzschild solution and the collapse of the lapse Phys. Rev. D 68124019

[42] Bizoń P, Rostworowski A and Zenginoğlu A 2010 Saddle-point dynamics of a Yang-Mills field on the exterior Schwarzschild spacetime Class. Quantum Grav. 27175003

[43] Bartnik R and McKinnon J 1988 Particlelike solutions of the Einstein-Yang-Mills equations Phys. Rev. Lett. 61 141-144

[44] Bizoń P 1990 Colored black holes Phys. Rev. Lett. $642844-2847$ 